\title{
Title
}

\section{Myriocin treatment of CF lung infection and inflammation: complex analyses for enigmatic lipids}

\section{Authors}

Anna Caretti ${ }^{1}$, Michele Vasso ${ }^{2}$, Fabiola Tecla Bonezzi ${ }^{1}$, Andrea Gallina ${ }^{1}$, Marco Trinchera ${ }^{3}$, Alice Rossi ${ }^{4}$, Raffaella Adami $^{1}$, Josefina Casas ${ }^{5}$, Monica Falleni ${ }^{1}$, Delfina Tosi ${ }^{1}$, Alessandra Bragonzi ${ }^{4}$, Riccardo Ghidoni ${ }^{1}$, Cecilia Gelfi ${ }^{2}$, Paola Signorelli ${ }^{*}$.

${ }^{1}$ Department of Health Sciences, University of Milan, Italy; ${ }^{2}$ Lita Institute, Segrate, University of Milan; ${ }^{3}$ Department of Medicine Clinical and Experimental, University of Insubria Medical School, Varese, Italy; ${ }^{4}$ Infections and Cystic Fibrosis Unit, San Raffaele Scientific Institute, Milan, Italy; ${ }^{5}$ Research Unit on Bioactive Molecules, Department of Biomedicinal Chemistry, Catalan Institute of Advanced Chemistry (IQAC/CSIC), Barcelona, Spain

*Correspondence to:

Dr. Paola Signorelli,

Biochemistry and Molecular Biology Laboratory, Department of Health Sciences, University of Milan, Via A.di Rudinì 8, 20142, Milan, Italy

Email: Paola.Signorelli@unimi.it Fax: ++390250323245

\begin{abstract}
Purpose. Our aim was to use quantitative and qualitative analyses to gain further insight into the role of ceramide in Cystic Fibrosis (CF). Sphingolipid ceramide is a known inflammatory mediator and its accumulation in inflamed lung has been reported in different types of emphysema, Chronic Obstructive Pulmonary disease and CF. CF is caused by a mutation of the chloride channel and associated with hyper-inflammation of the respiratory airways and high susceptibility to on-going infections. We have previously demonstrated that de novo ceramide synthesis is enhanced in lung inflammation and sustains $P$. aeruginosa pulmonary infection in a CF murine model. Methods. We used Liquid Chromatography and MALDI Imaging coupled with Mass Spectrometry, Confocal Laser Scan Microscopy and histology analyses to reveal otherwise undecipherable information. Results. We demonstrated that i) up-regulated ceramide synthesis in the alveoli is strictly related to alveolar infection and inflammation; ii) alveolar ceramide (C16) can be specifically targeted by nanocarrier delivery of the ceramide synthesis inhibitor Myriocin (Myr); iii) Myr is able to downmodulate pro-inflammatory lyso-PC, favouring an increase in anti-inflammatory PCs. Conclusions. We concluded that Myr modulates alveolar lipids milieu, reducing hyper-inflammation and favouring anti-microbial effective response in CF mouse model.
\end{abstract}

\section{Keywords}

Myriocin, Ceramide, Inflammation, Cystic Fibrosis, Mass Spectrometry. 


\section{Introduction}

Sphingolipids are a large family of molecules which play structural and signalling roles (Aguilera-Romero, 2014). Sphingolipid mediators have various involvements in human diseases (Brice 2011, Hammad 2011, Airola 2013, Maceyka 2014). Recent findings highlight the role of ceramide among sphingolipids in the pulmonary demise typical of chronic obstructive pulmonary disease (COPD) and Cystic Fibrosis (CF) (Grassme 2013, Petrache 2013). Gulbins and coworkers demonstrated that ceramide accumulates in CF airways and that amytriptiline, an inhibitor of the ceramidereleasing enzyme acid sphingomyelinase, reduces lung inflammation and bacterial infection in mice and in patients (Teichgraber 2008, Riethmuller 2009). We recently demonstrated that de novo ceramide synthesis also sustains lung inflammation and infection in CF (Caretti 2014) by using a murine CF model and pulmonary Pseudomonas aeruginosa infection to measure and pharmacologically target ceramide synthesis. Intratrachea injection of Myriocin (Myr), the inhibitor of Serine Palmitoyl Transferase (SPT), reduced inflammation and bacterial infection in CF mice. Although significant, the overall ceramide increase upon infection in CF mouse lung was not as striking as expected. We suspected that, as previously suggested (Becker 2010, Ulrich 2010), ceramide formation could be compartmentalized, and that quantitation on whole lung homogenates could underrate changes that occur in limited areas, such as the alveolar compartment. A second major concern raised by our studies considered the disentangling of ambiguities in the cause-effect relationship linking ceramide to inflammation and infection. Specifically, it is still unclear whether ceramide increases in response to a general inflammatory setting or whether it is a key mediator that drives acute response into chronic inflammation and consequent chronic infection. Our aim was to prove that ceramide accumulation, inflammation and bacterial colonization not only occur in the same lung region but also are strictly dependent on one another. We used different methods (Liquid Chromatography coupled with Mass Spectrometry, LCMS, MALDI Imaging, IMS and Confocal Laser Scan Microscopy, CLSM) to analyse the precise topologies of ceramide accumulation, $P$. aeruginosa and the inflammatory infiltrate, and we investigated the effect of ceramide synthesis inhibition in alveolar versus bronchial lung regions. We also explored the potential of solid lipid nanocarriers (SLN) (Caretti 2014), for in situ delivery of myriocin (Myr) in diseased lungs. SLNs have a solid lipid core, show strong interaction with membranes and are a long-lasting drug release system. We wanted to evaluate their pulmonary dispersal and to compare the topography of drug action with those of ceramide accumulation, inflammation, and consequent infection. Here we describe how we combined multiple in vitro sphingolipid detection methods to cope with all of the above issues. Using LC-MS, we precisely quantified single ceramide species and their metabolic derivatives (sphingomyelins and cerebrosides) in the whole lung. Using IMS, we were able to map the abundance of precise ceramide species onto histological images from lung sections. Furthermore, as this analytical approach allows for the simultaneous detection of all molecules in a sample across a broad mass and hydrophobicity range, we correlated ceramide changes with overall metabolic changes in other anti-inflammatory lipids, such as phosphatidylcholine (PC) (Treede 2007), and pro-inflammatory lipids such as lyso-PC (Yoder 2014), within specific tissue areas. Lastly, through immuno-staining and CLSM analyses, we confirmed the topography of ceramide within the lung and contrasted it with the location of bacterial infection. We concluded that the integrated use of these three different methodologies is able to reveal information that cannot be deciphered using just one single approach: i) up-regulated ceramide synthesis in the alveoli is strictly related to alveolar infection and inflammation; ii) alveolar ceramide (C16) can be specifically targeted by SLN delivery of the ceramide synthesis inhibitor Myr; iii) Myr is able to down-modulate the same acyl-chain bearing lyso-PC (C16 and C18) with a pro-inflammatory known action (Yoder 2014), favouring the increase in antiinflammatory PCs. 


\section{Methods}

Mice. Twelve weeks old gut-corrected CFTR deficient mice B6.129P2-Cftr ${ }^{\mathrm{tm} 1 \mathrm{UNC}} \mathrm{TgN}(\mathrm{FABPCFTR})$ (group KO) and congenic wild-type (Case Western Reserve University, Cleveland, Ohio, USA) (group WT) male and female mice were used. Mice were housed in filtered cages under specific-pathogen conditions and permitted unlimited access to food and water. Myriocin (Myr) administration was performed in the treated animals group, 24 hours prior to infection. Once a deep stage of anaesthesia with 2,2,2,-tribromoethanol (Avertin, Sigma-Aldrich, US) was reached, Myr loaded SLNs were intra-trachea instilled by means of MicroSprayer® Aerosolizer — Model IA1C, attached to "FMJ-250 High Pressure Syringe" (Penn-Century Inc., US). Control animals were treated with the corresponding empty SLNs. The total volume introduced in lungs was $50 \mu \mathrm{l}$, in mice of approximately $30 \mathrm{~g}$ each. This volume contained approximately $1,47 \mu \mathrm{g}$ of compound/mouse in SLN-saline solution. $24 \mathrm{~h}$ after treatment, animals (both KO and WT) were infected with 3-10 $\times 10^{5} \mathrm{CFU}$ of $P$. aeruginosa strain PAO1 planktonic cells, as previously described (Bragonzi 2010). At 18 h post-infection, mice were euthanized and lungs were explanted. Part of the samples were homogenized in $1 \mathrm{ml}$ of PBS containing protease inhibitors (Roche Italia, Italy) and homogenates divided in an aliquot for bacterial load counting (by serial dilution of homogenate sample in PBS and by plating on TSA) and an aliquot for lyophilization and lipids extraction for LC-MS analysis; part of the samples were embedded in a modified OCT and prepared for immunohistochemistry or IMS (Berry 2011). The other aliquot was serially diluted 1:10 in PBS and plated onto TSA plates to determine lung bacterial load. All experiments were performed with a minimum of five animals per group and a minimum of two experiments for each kind of investigation method (two experiments for IMS, two experiments for LSCM and 4 experiments for LCMS).

Myriocin loaded-solid lipid nanoparticles (SLN) used for mice treatment. Treatment of mice with Myr was achieved by using Myr-loaded SLNs (Nanovector srl, Italy) prepared as previously described. SLN loaded with drug were measured for Myr content and a $1 \mathrm{mM}$ Myr-SLN stock solution was prepared. This solution was diluted 1:12 in sterile saline and $50 \mu \mathrm{l}(1.47 \mu \mathrm{g}$ of Myr and $8 \%$ SLN) were used for each mouse administration in the airways.

LC-MS analysis. Sphingolipid extracts from both mice lungs and treated cells, fortified with internal standards $(N$ dodecanoylsphingosine, $N$-dodecanoylglucosylsphingosine, $N$-dodecanoylsphingosyl phosphorylcholine, C17sphinganine (0.2 nmol each) and C17-sphinganine-1-phosphate $(0.1 \mathrm{nmol})$, were prepared and analyzed as reported as reported.

Immunostaining and confocal microscopy analysis. Frozen lungs were embedded in OCT (Optimum Cutting Temperature-Compound, Leica Instruments, Nussloch, Germany), serial 5- $\mu \mathrm{m}$ thick sections were obtained in a cryomicrotome (Leica CM1510) and placed on silanized glass slides. The sections were dried for 3 min at room temperature, fixed in $4 \%$ buffered formalin for $45 \mathrm{~min}$ at $4{ }^{\circ} \mathrm{C}$, rinsed 2 times for $5 \mathrm{~min}$ in PBS, post-fixed with ethanol-acetic acid 2:1 (v/v) for $5 \mathrm{~min}$ at $-20^{\circ} \mathrm{C}$, rinsed twice for $5 \mathrm{~min}$ in PBS, boiled in $10 \mathrm{mM}$ citrate buffer at $\mathrm{pH}$ 6.0 for $10 \mathrm{~min}$, washed once in distilled water and three times in PBS, and finally used for immunofluorescence. After treatment with $10 \%$ normal goat serum, the sections were incubated overnight at $4{ }^{\circ} \mathrm{C}$ with a mouse anti-ceramide monoclonal antibody (Glycobiotech, Germany, diluted 1:100 in 1.5\% normal goat serum) and a rabbit polyclonal antiT7 directed against .P.aeruginosa (kindly provided by Dr.Pier, 1:50), followed by $1 \mathrm{~h}$ incubation with goat anti-mouse IgM fluorescein or with goat anti-rabbit IgG texas red-conjugated secondary antibody, respectively (Alexa, diluted 1:1000 in PBS). Total nuclei were counterstained with the karyophilic dye Hoechst 33258 (300 ng/ml, Sigma) for 3 min at room temperature, in the dark, followed by rinsing twice in PBS. Sections on glass slide were then mounted in a 10:1 
glycerol-PBS medium, $\mathrm{pH} 8.5$, containing $0.1 \%$ p-phenylenediamine as anti-quenching agent. Confocal imaging was performed on a Leica SP2 confocal microscope (Leica Microsystems), using a 40X (NA: 1.25) oil immersion objective and 405, 488 and $633 \mathrm{~nm}$ lasers. Images were collected using sequential acquisition process in order to reduce spectral crosstalk between channels and stacks collapsed into projection images. Offline handling, segmentation and geometric density analysis was developed with LCS software (version 2.6.1; Leica Microsystems).

Histochemistry. Both lungs from infected KO and WT mice, either pre-treated with SLN-Myr or untreated (SLNempty) where fixed in buffered formalin and routinely processed for histopathological evaluation. Briefly, lungs were sectioned, and paraffin-included. Twenty serial 3-micron thick sections were cut at a microtome; slides at different levels (level 1,11 and 20) were stained with hematoxylin and eosin to have a comprehensive estimation of the distribution of inflammatory infiltrate in all compartments. Slides were observed at a Leika light microscope. Image acquisition was performed by the NanoZoomer-XR C12000 series (Hamamatsu Photonics K.K., Tokio, Japan.). The site of inflammation (bronchial and bronchiolar compartment- intraparietal and endoluminal- and alveoli) and its intensity (low: + , medium: ++ and high: +++ ) were considered. In each case a median value of flogistic reaction on the three compartments was considered.

MALDI-IMS analysis. Lungs from infected CF and WT, either pre-treated or not with SLN-Myr, where explanted 24 $\mathrm{h}$ after infection fixed, sliced at a thickness of $12 \mathrm{um}$ with a cryostat at $20^{\circ} \mathrm{C}$, mounted onto a ITO glass and DHB matrix was overlayed by sublimation onto the samples using a sublimation apparatus (ACE Glass) for 20 min at 50 mTorr pressure at $180^{\circ} \mathrm{C}$. The MALDI-IMS analyses were performed by Ultraflex III time-of-flight mass spectrometer (Bruker Daltonics) with a SmartBeam laser operating at $200 \mathrm{~Hz}$ in positive linear mode using FlexControl 3.0 and FlexImaging 2.1 software packages (Bruker Daltonics). Ions were detected in the 480-900 m/z mass range with a sampling rate of $2 \mathrm{GS} / \mathrm{s}$. The lateral resolution for MALDI-IMS was set to $50 \mu \mathrm{m}$, and a total of 100 laser shots were accumulated per pixel at constant laser power. Two-dimensional ion intensity maps and average spectra were created by FlexImaging 2.1 software (Bruker Daltonics). In order to identify differentially expressed peaks, tandem mass spectrometry (MS/MS) analysis was performed using LIFT-TOF/TOF mode and the product ions characteristic head-group of the lipid was used to classify the lipids of interest.

Statistical Analyses. Fluorescence quantitation and statistical evaluation. On the basis of the histological staining, regions of interest (ROIs) were defined in the tissue section using the FlexImaging 2.1 software (Bruker Daltonics), ROIs containing the same number of spectra (around 70) were created. Statistical analyses were carried out using the ClinProTools 2.2 software (Bruker Daltonics) and, during the preprocessing steps, the extracted mass spectra were internally recalibrated, normalized on their total ion and corrected by a baseline subtraction. An average spectrum created for each class was used for peak picking and to define integration ranges to be used to obtain the area of each peak (m/z species) in each single spectrum. A non paramentric Wilcoxon/ Kruskal-Wallis test was performed, with a significance cutoff point of $\mathrm{p}$-value $<0.05$. A receiver operator characteristic (ROC) analysis was performed to highlight peaks discriminating the compared classes (area under curve (AUC) > 0.800). LC-MS and IMS, histochemistry images statistical evaluation. Most of the analyses, comparing treated and untreated, were performed by unpaired two tailed T student test., $p<0,05$. In some cases (reported in figure legend), Anova one way test was performed, $p<0,05$, followed by post-test Bonferroni. 


\section{Results}

Ceramide levels correlate with $\boldsymbol{P}$. aeruginosa lung colonies. Wild type (WT) and CFTR knock out (KO) mice were infected by intratracheal nebulization of $3 \times 10^{\wedge} 5$ P. areuginosa CFU. A group of mice received $50 \mu 1$ containing a suspension of Myr loaded SLN (1.47 $\mu \mathrm{g}$ /mouse of the compound), 24 hours prior to infection. Mice were sacrificed 24 hours after infection and lung was explanted for analyses. We measured total ceramide mass and P. aeruginosa lung colonies both in WT and KO infected mice, either treated with Myr or untreated. We observed a strong direct correlation between lung ceramide content and total pulmonary bacterial colonies (statistical significance $\mathrm{p}=0.0164$ ), showing that ceramide accumulation is closely associated with infection (Figure 1). This occurred both in WT and KO mice although, as regards significant differences in absolute values, KO mice were more severely infected and showed higher ceramide levels than wild type (Caretti 2014).

Specific ceramide pools are increased in KO mice and modulated by Myriocin. The analyses of lung homogenate revealed similar ceramide species profiles both in WT and KO mice, although a few species are slightly but reproducibly higher in $\mathrm{KO}$ (mean value of total ceramide picomoles per milligram of protein in WT mice $839.3 \pm 61$ versus $\mathrm{KO}$ mice $1058.2 \pm 49, \mathrm{p}=0.038$ ). Treatment with $\mathrm{SLN}-\mathrm{Myr}$ reduced almost all ceramide species, both in WT and KO mice (Figure 2A). Specifically, higher significance in Myr induced reduction was observed in the most representative ceramide species (Figure 2A), (as C16 ceramide, mean \pm SD: KO untreated $178 \pm 16,9$ versus treated $97.9 \pm 10.2$; saturated C24-ceramide, KO treated: $217 \pm 49.6$ versus untreated $509.8 \pm 4$ 9.2; mono-unsaturated C24:1ceramide, KO treated $97.9 \pm 10.2$ versus untreated $178.6 \pm 16.9$ ). Although a minor species, C18-ceramide was significantly reduced by half (mean $\pm \mathrm{SD}$ : KO treated $12.6 \pm 1.5$ versus untreated $23.8 \pm 3.6$ ). These observations suggest that KO mouse lung, upon infection, undergoes an increased synthesis of ceramide, mainly affecting C16 and C24 saturated/mono-unsaturated ceramides, compared to WT mouse lung. This is also supported by the previously reported increase of serine palmitoyl transferase expression in the same KO versus WT infected mice (Caretti 2014). No significant variability was observed in the sphingomyelins pools (Figure 2B), whereas mono-glycosylated ceramides were affected by Myr treatment, with the C16 and C18 acyl-chain species being significantly reduced in KO treated versus untreated lungs (Figure 2C). When considering the percentage change in ceramides in the KO and WT mice, it is interesting to note that the Myr-induced reduction in the percentage of C16, C18 and C24 ceramides is significantly higher in KO than in WT mice (Figure 2D), indicating that de novo ceramide synthesis is enhanced and drug treatment is effective in CF murine model lung.

Inflammatory lyso-PC is reduced by Myriocin in infected mice. PC is a known anti-inflammatory lipid and oral phosphatidylcholine alleviates inflammatory activity caused by ulcerative colitis (Stremmel 2005). Lyso-PC is obtained through the action of cPLA2 on PC and exerts pro-inflammatory actions. Choline is shared among sphingolipids and glycerophospholipids, being incorporated into SM and PC, and ceramide is able to activate cPLA2 (Sato et al. 2009, Kitatani et al. 2000, Huwiler et al 2001). We observed a slight but consistent increase in PC, with a particularly high mass in cells and tissue, and a significant drop in lyso-PC (mean value of lyso-PC picomoles per milligram of protein \pm SD: value $5167 \pm 99$ vs $4393 \pm 200$ ) in infected and Myriocin-treated KO mice versus infected and untreated mice (Figure 3A and B). Since PC mass is particularly high in tissue and cells, a slight increase must be taken into account. PC can be metabolized in different by-products, so LysoPC basal levels are normally much lower and its increase along with an increase in ceramide synthesis indicates that, in $\mathrm{KO}$ mice, it is able to modulate glycerophospholipid pools and promote the formation of pro-inflammatory lyso-PC. 
Localization of ceramide and $\boldsymbol{P}$. aeruginosa within the lungs. In order to understand the meaning of ceramide

increase, we investigated the topology of its accumulation within the lung and co-related it to P. aeruginosa localization using confocal laser scanning microscopy. 24 hours after infection or Myr-treatment and infection, the lungs of KO and WT mice were explanted and stained with anti-ceramide antibodies and with anti-P. aeruginosa antibodies. Myr treatment succeeded in reducing both ceramide and P. aeruginosa PAO1 strain in the alveolar and bronchial regions (Figure 4A and 4B). The quantitation of immunofluorescence, deriving from DAPI positive cells only, provided us with confirmation of LC-MS data and allowed us to discriminate between alveolar and bronchial ceramides. We measured an increased ceramide signal in infected KO versus WT lungs and a slight but significant increase in $P$. aeruginosa infection in KO lungs (Figure 4C), in agreement with the LC-MS quantitation of ceramides and the CFU count of Figure 1We also found a higher accumulation of ceramide in the alveolar areas (Figure 4 A, infected only WT panel A and KO infected only panel G), an overlap of $P$. aeruginosa in the alveolar regions with ceramides (Figure 4A, infected only WT panel $\mathrm{C}$ and KO infected only panel I), and a reduction in $P$. aeruginosa (Figure 4A WT infected and treated, panel E, KO infected and treated, panel K) and a dramatic reduction in ceramide in these areas (Figure 4A WT infected and treated, panel D, KO infected and treated, panel J) following Myr treatment.

\section{Ceramide and immune infiltrate located in the alveolar areas.}

In our previous publication, we demonstrated that Myr is able to reduce the transcription of inflammatory mediators by evaluating the whole lung homogenate of KO and WT mice (Caretti 2014), although we were unable to obtain significant differences in the inflammatory cells present in BAL of treated and untreated mice. In order to clarify the role of Myr in the acute immune response, we used light microscopy to evaluate the presence of infiltrate in infected KO and WT mice, either treated with Myr or untreated. We noticed a basal difference between infected KO and WT mice, with the former exhibiting a higher and more widespread infiltrate, especially in the lower airways (bronchioli and alveoli, Figure 5 a, b, c, g, h, i). We observed a dramatic reduction of inflammatory cells in Myr treated lungs (Figure 5 WT mice d, e, f and KO mice 1, m, n), which was particularly evident in the KO alveolar areas (n). In treated mice, flogosis showed a "patchy" pattern, with almost healthy areas near unhealthy zones; where groupss of neutrophils and, to a lesser extent, macrophages were commonly detected. In untreated mice, the same inflammatory reaction involved all the compartments studied equally. The results are summarized in table 1 . These observations confirm that localized control of sphingolipid metabolism, obtained through alveolar Myr delivery, is highly effective in lung infection and inflammation. We concluded that SLN drug delivery is highly suitable for alveolar targeting.

\section{Quantification and topology in vivo of single ceramide species.}

In order to confirm the localization of ceramide and to obtain its quantitative fluctuation within tissue, we performed a MALDI Imaging analysis. Lungs from infected CF and WT mice, some of which pre-treated with SLN-Myr, were explanted $24 \mathrm{~h}$ after infection. They were then fixed, sliced and embedded into matrix for MALDI-TOF analysis. Utilizing hematoxylin and eosin-stained images, ROIs of lung sections were defined in each MALDI-IMS experiment and peak areas within the $480-900 \mathrm{~m} / \mathrm{z}$ range were then submitted to statistical analysis. Slices were also scanned and stained for histochemistry, and the images were overlapped. As far as ceramide species were concerned, a $538.20 \mathrm{~m} / \mathrm{z}$ peak, corresponding to C16-ceramide, was detected in all samples, and the peak level was reduced using SLN-Myr treatment in both WT and KO mice, in accordance with UPLC-TOF data (Fig. 6A). Moreover, the extracted-ion MALDI image of the $538.20 \mathrm{~m} / \mathrm{z}$ peak, indicates a relatively even intensity within the lung parenchyma (Fig 6B), while SLN-Myr treatment reduces C16 ceramide in the alveolar region more than in the bronchial region, both in WT and KO 
mice (Figure 6 panel A versus C). To better characterize sphingolipid differences related to treatment in WT and KO mice and find other species capable of discriminating these groups, a statistical analysis of spectra obtained through MALDI-IMS experiments was performed using ClinProTools software. In particular, a ROC analysis was performed to highlight discriminating peaks (AUC > 0.800). By comparing WT Myr-untreated versus treated, we found 30 differentially expressed peaks ( 12 increased and 18 decreased after pharmacological treatment) with $\mathrm{p}<0.5$ and $\mathrm{CV}<20$ $\%$ (table 1), nine of which were identified by MALDI-LIFT-MS/MS analysis directly on tissue slices (Supplementary figures 1-10). The whole analysed slice is reported in figure 7A. The positive-ion collision-induced dissociation (CID) analysis of $\mathrm{m} / \mathrm{z} 496.42$ and 524.52 peaks identified C16 and C18 lyso-PC, respectively (Figure 7B and C). As found for C16-Cer, these ions had lower intensities in treated samples than in those that were not treated and were evenly distributed in the lung slice (Fig 7B and C). On the other hand, peaks at $m / z, 732.78,754.72,780.67,782.68,804.58$ and 806.59, corresponding to 32:1-PC (M+1), 32:1-PC (M+ Na), 34:2-PC (M+ Na), 34:1-PC (M+ Na), 36:4-PC (M+ Na) and 38:6-PC (M+1), respectively, were higher in treated samples. In addition, 32:1-PC (M+1), 32:1-PC (M+ Na) and 34:1-PC (M + Na) showed a distribution very similar to C16-Cer and lysoPC species (Fig 7D, E and G), whereas the signal corresponding to 34:2-PC (M + Na) was evenly distributed across the lung slice with a slight increment in the bronchus region (Fig. 7F). By contrast, MALDI analysis of 36:4-PC $(\mathrm{M}+\mathrm{Na})$ and 38:6-PC $(\mathrm{M}+1)$ indicated that these phospholipid molecular ions were highly abundant along the bronchus and were also present in the alveolar region (Fig. 7H and I). Regarding the comparison between untreated versus treated KO mice, 14 differentially expressed peaks (7 increased and 7 decreased after pharmacological treatment) with $\mathrm{p}<0.5$ and $\mathrm{CV}<20 \%$ were detected (table 2), and five of them were identified by MALDI-LIFT-MSMS analysis (Supplementary figure 1). In particular, peaks at $\mathrm{m} / z$ 732.78, 754.72, 760.78, 770.69 and 780.78, corresponding to 32:1-PC (M+1), 32:1-PC (M + Na), 34:1-PC (M + 1), 32:1PC (M $+\mathrm{K})$ and 34:2-PC $(\mathrm{M}+\mathrm{Na})$, respectively, were augmented in treated samples. Identified phosphatidylcholines showed even distribution along the tissue slice with the exception of 34:2-PC, which was only slightly expressed in the bronchus (Fig.8). Overall, data obtained using IMS demonstrate that: i) C16-ceramide is more abundant in alveolar areas and ii) C16-ceramide and lyso-PCs, the latter being known pro-inflammatory mediators, are reduced with Myr treatment, favouring an increase in PC (see hypothesis scheme, Figure 9).

\section{Discussion}

The study of sphingolipid mediators is quite challenging due to the large number of bioactive molecules metabolically converting one into another, their different signalling in relation to subcellular distribution or extracellular release, their subtle switches from homeostatic concentration to accumulation and related "alarm-stress" signals and, last but not least, their diverse level of hydrophobicity, requiring appropriate extraction from samples. This document aims to embrace different analytical techniques and integrate all the data obtained, in order to achieve conclusions that cannot be fully deduced from a single analysis. After decades of sphingolipid evaluation using labelled precursors, in vitro or in vivo, the arrival of LC-MS analysis for lipidomic studies allows accurate quantitation and, moreover, the distinction among all different species. The evaluation of single species fluctuations has opened up the possibility to define actions and metabolic routes for previously under-estimated molecules such as C18-versus C16-ceramide (Karahatay 2007), dihydroceramides versus ceramides (Fabrias 2012), as well as sphingosine-1-phosphate versus sphingosines (Takabe 2008) and ceramide-1-phosphate versus ceramide (Gomez-Munoz 2006). Despite representing a step forward, this method cannot consider the scenario of sphingolipid modulation, which is orchestrated by environmental stimuli within a single body. Therefore, sphingolipid modulation can occur as a result of a given stress but not elsewhere, such as in 
the brain at the outset of Alzheimer's or Parkinson's (Fabelo 2011, Fiedorowicz 2014, Panchal 2014), or in lung infection and inflammation (Ulrich 2010). Research requires the identification of the location of sphingolipid action both for the evaluation of their role and in order to approach pharmacological targeting. Immunocytochemical studies have been applied for the location of ceramides within tissue. In 1992, an interesting article by Hannun's group was published in JLR, assessing and questioning the specificity and the reliability of different ceramide antibodies (Cowart 2002). Being mostly membrane embedded, it is quite difficult to detect the specific binding of ceramide antibody, although several authors reported successful use of this tool. IMS, an innovative recent application of MALDI imaging to MS, can trace lipid variation directly on tissue slides without extraction, thus maintaining all the information related to their location. Although hard to consider as a quantitative analysis, IMS is a highly promising tool for research on sphingolipids roles in vivo. One of our main unanswered questions was "how is ceramide accumulation related to lung infection?". On one hand, ceramide deriving from increased plasma membrane sphingomyelinases activity, previously demonstrated by other authors (Riethmuller 2009), can be a consequence of the engagement of pattern-recognition or inflammatory mediator receptors, within the context of a self-resolving inflammatory response triggered by acute bacterial infection. On the other, the increase of the rate-limiting enzyme of de novo sphingolipid synthesis, recently reported by us and by others in lung inflammation (Petrache 2013, Caretti 2014), is probably due to a metabolic switch of the airways' epithelia, which is partially responsible for the chronic inflammation that characterizes CF-diseased lung and encourages infection rather than opposing it. In our CF mice model, we observed an anti-inflammatory effect of Myr associated to a decrease in bacteria lung colonization. We demonstrated that Myr was more effective in KO than WT mice (Figure 2D). We concluded that ceramide increase is particularly enhanced in KO mice, via its de novo synthesis, and Myr is specifically effective in counteracting sphingolipid-altered metabolism in CF. In support of this idea, thanks to precise quantitation offered by LC-MS and with the broad insight offered by IMS, we found that other pro-inflammatory lipids are up-regulated at the same time as ceramide, suggesting a comprehensive lipid dysmetabolic reaction to infection. It is important to note that the pharmacological inhibition of ceramide synthesis is able to regulate other inflammatory lipids, by reducing known inflammatory lyso-PC production (Yoder 2014) and increasing the accumulation of anti-inflammatory PC (Treede 2007). Lyso-PC derives from phospolipases activity and it was previously reported that ceramide is able to activate cytosolic PLA2 (Sato et al. (1999), Kitatani et al. (2000), Huwiler et al. 2001), possibly creating a metabolic loop between the two inflammatory molecules. It is interesting to note that species containing saturated $\mathrm{C} 16$ (and C18) acyl chains, previously reported to have specific inflammatory activity (Yoder 2014), are significantly reduced both in ceramides and lyso-PC families, suggesting that Myr treatment may control palmitate availability for other metabolic reactions, such as the formation of anti-inflammatory PC, thanks to the down-modulation of stress-induced ceramide synthesis,. These data highlight a central role for ceramide metabolism in the metabolism of inflammatory lipids and suggest that the rate of de novo sphingolipid synthesis is carefully balanced and plays a sentinel role in the response to stress and in the switch to a pathological state. This is an important concept that requires exploration in other chronic inflammatory settings with the aim of being considered as a therapeutic target. Moreover, our data confirm the idea that bacterial infection from the upper airways reaches the inner lungs and that there is a significant burden of alveolar epithelia favoured by local ceramide accumulation, and consequent recruitment of inflammatory cells. The demonstrated phenotype switch in the infectious population and the acquisition of resistance mechanisms (Bragonzi 2009) can be associated with bacterial flow from the upper to lower airways and, lastly, the alveoli (McAdam 2011). It is mandatory to approach drug delivery methods that ensure the targeting of the alveolar region. Myr treatment evidenced an overall reduced inflammation with pro-resolving patchy 
granulocyte aggregates and no inflammation-related extensive damage of the alveolar structure. Our Solid Lipid

Nanoparticles demonstrated a marked ability to reach the alveolar compartment and to efficaciously release the drug.

Acknowledgments. The authors thank B. Tümmler (Klinische Forschergruppe, Medizinische Hochschule Hannover, Germany) for supplying the $P$. aeruginosa AA2 clinical strain and G. Pier for the rabbit antiserum specific for P.aeruginosa. Financial and administrative support from Italian Cystic Fibrosis Research Fundation is acknowledged (FFC \#20-2013). The University of Milan PhD program in Molecular Medicine is acknowledged. Fondazione Roma is acknowledged for the financial support in Myriocin-SLN and post-doctoral fellowship.

\section{References}

Aguilera-Romero A, Gehin C, Riezman H (2014) Sphingolipid homeostasis in the web of metabolic routes. Biochim Biophys Acta 1841(5):647-56. doi: 10.1016/j.bbalip.2013.10.014

Airola MV, Hannun YA (2013) Sphingolipid metabolism and neutral sphingomyelinases. Handb Exp Pharmacol:57-76. doi: 10.1007/978-3-7091-1368-4_3

Becker KA, Tummler B, Gulbins E, Grassme H (2010) Accumulation of ceramide in the trachea and intestine of cystic fibrosis mice causes inflammation and cell death. Biochem Biophys Res Commun 403(3-4):368-74. doi: 10.1016/j.bbrc.2010.11.038

Berry KA, Li B, Reynolds SD, Barkley RM, Gijon MA, Hankin JA, Henson PM, Murphy RC (2011) MALDI imaging MS of phospholipids in the mouse lung. Journal of lipid research. 52(8):1551-60. doi: 10.1194/jlr.M015750

Bragonzi A, Paroni M, Nonis A, Cramer N, Montanari S, Rejman J, Di Serio C, Döring G, Tümmler B (2009) Pseudomonas aeruginosa microevolution during cystic fibrosis lung infection establishes clones with adapted virulence. Am J Respir Crit Care Med 15;180(2):138-45. doi: 10.1164/rccm.200812-1943OC

Bragonzi A (2010) Murine models of acute and chronic lung infection with cystic fibrosis pathogens. Int J Med Microbiol 300(8):584-93. doi: 10.1016/j.ijmm.2010.08.012

Brice SE, Cowart LA (2011) Sphingolipid metabolism and analysis in metabolic disease. Adv Exp Med Biol 721:1-17. doi: 10.1007/978-1-4614-0650-1_1

Caretti A, Bragonzi A, Facchini M, De Fino I, Riva C, Gasco P, Musicanti C, Casas J, Fabrias G, Ghidoni R, Signorelli $\mathrm{P}$ (2014) Anti-inflammatory action of lipid nanocarrier-delivered myriocin: therapeutic potential in cystic fibrosis. Biochim Biophys Acta 1840(1):586-94. doi: 10.1016/j.bbagen.2013.10.018

Cowart LA, Szulc Z, Bielawska A, Hannun YA (2002) Structural determinants of sphingolipid recognition by commercially available anti-ceramide antibodies. J.Lipid Res. 43(12):2042-8.

Fabelo N, Martín V, Santpere G, Marín R, Torrent L, Ferrer I, Díaz M (2011) Severe alterations in lipid composition of frontal cortex lipid rafts from Parkinson's disease and incidental Parkinson's disease. Mol Med 17(9-10):1107-18. doi: $10.2119 /$ molmed

Fabrias G, Muñoz-Olaya J, Cingolani F, Signorelli P, Casas J, Gagliostro V, Ghidoni R (2012) Dihydroceramide desaturase and dihydrosphingolipids: debutant players in the sphingolipid arena. Prog Lipid Res 51(2):82-94. doi: $10.1016 / \mathrm{j}$

Fiedorowicz A, Prokopiuk S, Zendzian-Piotrowska M, Chabowski A, Car H (2014) Sphingolipid profiles are altered in prefrontal cortex of rats under acute hyperglycemia. Neuroscience 256:282-91. doi: 10.1016/j.neuroscience 
Ghidoni R, Caretti A, Signorelli P Role of Sphingolipids in the Pathobiology of Lung Inflammation. Mediators Inflamm. 2015:487508. doi: 10.1155/2015/487508

Grassme H, Riethmuller j, Gulbins E (2013) Ceramide in cystic fibrosis. Handb Exp Pharmacol:265-274. doi: 10.1007/978-3-7091-1511-4_13

Gómez-Muñoz A (2006) Ceramide 1-phosphate/ceramide, a switch between life and death. Biochim Biophys Acta 1758(12):2049-56

Hammad SM (2011) Blood sphingolipids in homeostasis and pathobiology. Adv Exp Med Biol 721:57-66. doi: $10.1007 / 978-1-4614-0650-144$

Ji JE, Kim SK, Ahn KH, Choi JM, Jung SY, Jung KM, Jeon HJ, Kim DK (2011) Ceramide induces serotonin release from RBL-2H3 mast cells through calcium mediated activation of phospholipase A2. Prostaglandins \& other lipid mediators 94(3-4):88-95. doi: 10.1016/j.prostaglandins.2011.01.001

Karahatay S, Thomas K, Koybasi S, Senkal CE, Elojeimy S, Liu X, Bielawski J, Day TA, Gillespie MB, Sinha D, Norris JS, Hannun YA, Ogretmen B (2007) Clinical relevance of ceramide metabolism in the pathogenesis of human head and neck squamous cell carcinoma (HNSCC): attenuation of C(18)-ceramide in HNSCC tumors correlates with lymphovascular invasion and nodal metastasis. Cancer Lett 18;256(1):101-11

McAdam PR, Holmes A, Templeton KE, Fitzgerald JR (2011) Adaptive evolution of Staphylococcus aureus during chronic endobronchial infection of a cystic fibrosis patient. PLoS One 6(9):e24301. doi: 10.1371/journal.pone.0024301

Maceyka M, Spiegel S (2014) Sphingolipid metabolites in inflammatory disease. Nature 510:58-67 doi: $10.1038 /$ nature13475

Munoz-Olaya JM, Matabosch X, Bedia C, Egido-Gabas M, Casas J, Llebaria A, Delgado A, Fabrias G (2008) Synthesis and biological activity of a novel inhibitor of dihydroceramide desaturase. ChemMedChem 3(6):946-53. doi: $10.1002 / \mathrm{cmdc}$

Nakamura H, Murayama T (2014) Role of sphingolipids in arachidonic acid metabolism. Journal of pharmacological sciences 124(3):307-12

Panchal M, Gaudin M, Lazar AN, Salvati E, Rivals I, Ayciriex S, Dauphinot L, Dargère D, Auzeil N, Masserini M, Laprévote O, Duyckaerts C (2014) Ceramides and sphingomyelinases in senile plaques. Neurobiol Dis 65:193-201. doi: 10.1016/j.nbd.2014.01.010

Petrache I, Petrusca DN (2013) The involvement of sphingolipids in chronic obstructive pulmonary diseases. Handb Exp Pharmacol (216):247-64. doi: 10.1007/978-3-7091-1511-4_12

Riethmuller J, Anthonysamy J, Serra E, Schwab M, Doring M, Gulbins E (2009) Therapeutic efficacy and safety of amitriptyline in patients with cystic fibrosis. Cell Physiol Biochem 24(1-2):65-72. doi: 10.1159/000227814

Stremmel W, Merle U, Zahn A, Autschbach F, Hinz U, Ehehalt R (2005) Retarded release phosphatidylcholine benefits patients with chronic active ulcerative colitis. Gut 54(7):966-71

Takabe K, Paugh SW, Milstien S, Spiegel S (2008) "Inside-out" signaling of sphingosine-1-phosphate: therapeutic targets. Pharmacol Rev 60(2):181-95. doi: 10.1124/pr.107.07113

Teichgraber V, Ulrich M, Endlich N, Riethmuller J, Wilker B, De Oliveira-Munding CC, van Heeckeren AM, Barr ML, von Kurthy G, Schmid KW, Weller M, Tummler B, Lang F, Grassme H, Doring G, Gulbins E (2008) Ceramide accumulation mediates inflammation, cell death and infection susceptibility in cystic fibrosis. Nat Med 14(4):382-91. doi: $10.1038 / \mathrm{nm} 1748$.

Treede I, Braun A, Sparla R, Kuhnel M, Giese T, Turner JR, Anes E, Kulaksiz H, Fullekrug J, Stremmel W, Griffiths G, Ehehalt E (2007) Anti-inflammatory effects of phosphatidylcholine. J Biol Chem 282:27155-64 
Ulrich M, Worlitzsch D, Viglio S, Siegmann N, Iadarola P, Shute JK, Geiser M, Pier GB, Friedel G, Barr ML, Schuster A, Meyer KC, Ratjen F, Bjarnsholt T, Gulbins E, Doring G (2010) Alveolar inflammation in cystic fibrosis. J Cyst Fibros 9(3):217-27. doi: 10.1016/j.jcf.2010.03.001

Yoder M, Zhuge Y, Yuan Y, Holian O, Kuo S, van Breemen R, Thomas LL, Lum H (2014) Bioactive lysophosphatidylcholine 16:0 and 18:0 are elevated in lungs of asthmatic subjects. Allergy Asthma Immunol Res 6(1):61-5. doi: 10.4168/aair.2014.6.1.61

\section{Legends}

Table 1. A sum up of the histochemistry analyses result is provided in the the table: IP,intraparietal; EL, endoluminal; $\mathrm{P}$, patchy; D, diffuse; :+low; ++ medium; +++ high inflammatory infiltrate.

.Table 2. Differentially expressed peaks $(\mathrm{p}<0.05)$ obtained by comparison between untreated and SLN-Myr treated wild-type mice. For each peak p-value obtained adopting a non-parametric test (Wilcoxon test), class average areas, $\mathrm{CV}, \mathrm{ROC}$ value and assignment are indicated.

Table 3. Differentially expressed peaks ( $\mathrm{p}<0.05)$ obtained by comparison between untreated and SLN-Myr treated knock-out mice. For each peak p-value obtained adopting a non-parametric test (Wilcoxon test), class average areas, $\mathrm{CV}, \mathrm{ROC}$ value and assignment are indicated

Figure 1. Correlation of total ceramide levels with lung infection. Total ceramides were measured by LC-MS and CFU counts were collected from lung homogenates. Data were obtained from KO and WT mice either untreated (SLNempty) or treated with SLN-Myr. Three separate experiments were conducted. Linear regression afforded a $\mathrm{P}$ values of 0.0164

Figure 2. LC-MS quantification of different acyl species ceramides (A), sphingomyelins (B) and mono-glycosylated ceramides (glucose or galactose) (C), in Myriocin treated (SLN-Myr) versus untreated (SLN-empty) KO and WT total lung homogenates. Sphingolipids picomoles are normalized onto proteins milligrams per sample. Unpaired student $t$ test two tails was performed, between Myriocin treated versus untreated. $*=p<0.05, * *=p<0.01$. Panel (D) shows the percentage of the three most representative ceramides (bearing C16:0 or C18:0 or C24:0 fatty acid acyl chain). Untreated KO or WT were considered as $100 \%$ of each ceramide specie (gray bars), and the percentages of ceramides were calculated in Myriocin treated lung homogenates. Data shows a significantly higher reduction of ceramides in KO-treated lung homogenate than in WT. Anova test was performed among $g_{*}$ all groups $(\mathrm{p}<0,0001)$, followed by Bonferroni test in KO versus WT single chains ceramides $* * *=p<0,001$

Figure 3. LC-MS quantification of phosphatidylcholine (PC) (A) and lyso-phosphatidylcholine (lyso-PC) (B) in total lung homogenates from Myriocin treated (SLN-Myr) versus untreated (SLN-empty) infected KO and infected WT mice. Lipids picomoles are normalized onto proteins milligrams per sample. Unpaired student t test two tails was performed, between Myriocin treated versus untreated; *, p<0,05.

Figure 4. Ceramide and PAO1 localization in the lung. Confocal laser scan images, most representative of alveolar (A) and bronchial (B) areas .Left vertical lane: anti-ceramide fluorescence (green); middle vertical lane anti-P.aeruginosa fluorescence(red); right vertical lane overlapping of the two staining. a,b,c) WT mice untreated (SLN-empty); d,e,f) WT mice treated with SLN-Myr; g,h,i) KO mice untreated (SLN-empty); 1,m,n) KO mice treated with SLN-Myr. Images are stack average projections, bar $=50 \mu \mathrm{m}$. C) Total lung Ceramide content and PAO1 infection (immunofluorescence quantitation). Statistical evaluation was performed by Unpaired t-test: *, p<0,05, ceramide in $\mathrm{KO}$ treated versus $\mathrm{KO}$ untreated; \#, p<0,05, PAO1 in KO untreated versus WT untreated. D) Ceramide content (immunofluorescence 
quantitation) in alveolus versus broncus, in infected or infected and myriocin treated KO mice. Statistical evaluation was performed by Unpaired t-test: *, p<0,05, ceramide in $\mathrm{KO}$ treated versus $\mathrm{KO}$ untreated.

Figure 5. Lung histology. a, b, c: WT mice infected/ untreated; d, e, f: WT mice infected/treated with Myriocin. g, h, i : KO mice infected/untreated; 1, m, n: KO mice infected/treated with Myriocin. WT empty. a): bronchial lumina and walls are free from inflammatory elements. b): moderate inflammatory cells are detected both in the lumen and in the walls of bronchiole. c): diffuse moderate inflammatory elements invading alveoli, even in sub-pleural areas. d): no inflammatory infiltrate is detected in or around large bronchi. e): sporadic inflammatory cells are observed in the bronchiolar walls and only rarely, in the bronchiolar lumen. f): Very weak inflammatory infiltrate in the alveolar compartment; $\quad \mathbf{g} \& \mathbf{h}$ ) very dense inflammatory infiltrate (black arrows) is easily visible in the lumen of large ( $\mathbf{g}$ ) and small (h) airways, as well as in the surrounding parenchima.i): heavy flogistic infiltrate diffusely infiltrate lung alveoli; 1): inflammatory infiltrate in the walls of a large broncus and in peri-bronchial tissues (arrow); no flogistic elements are observed in the lumen (L). m): inflammatory elements are detected in the bronchiolar walls and at a lesser extent, in the lumen (L). A weak to moderate infiltrate is also observed in the alveolar compartment in a pathway pattern. n): Weak patchy inflammatory alveolar infiltrate reaches the sub-pleural areas.

Figure 6. Ceramide distribution in the lung. MALDI Imaging analysis of $538.2 \mathrm{~m} / \mathrm{z}$ peak, corresponding to C16:0 ceramide. (A) Box plots indicating the expression of 538,2 m/z peak in the lung tissue of wild type (WT) and CF (KO) mice, either untreated (SLN-empty) or myriocin treated (SLN-Myr) (B) Distribution of $538.2 \mathrm{~m} / \mathrm{z}$ peak (blue color) in lung tissue of WT and KO mice with and without pharmacological treatment. (C) Box plots indicating the expression of $538,2 \mathrm{~m} / \mathrm{z}$ peak in wild type and knock-out mice with and without myriocin treatment only in the bronchial area. For bronchi and alveoli distinction, hematoxylin-eosin staining was obtained on the same slices and MALDI image was overlapped to histochemistry image.

Figure 7. MALDI Imaging analysis of differentially expressed and identified peaks in untreated versus SLN-Myr treated wild-type mice lung. Distribution, box plots and ROC curve of (B) lysoPC (16:0) (C) lysoPC (18:0) (D) PC (32:1), (E) PC (32:1) + Na, (F) PC (34:2) + Na, (G) PC (34:1) + Na, (H) PC (36:4) + Na and (I) PC (38:6) are shown.

Figure 8. MALDI Imaging analysis of differentially expressed and identified peaks in untreated versus SLN-Myr treated KO mice lung. Distribution, box plots and ROC curve of (B) PC (32:1), (C) PC (32:1) + Na, (D) PC (34:1), (E) $\mathrm{PC}(32: 1)+\mathrm{K}$, and $(\mathbf{F}) \mathrm{PC}(34: 2)+\mathrm{Na}$ are shown.

Figure 9. Scheme: hypothesis of Myr regulating inflammatory lipids. Red, inflammatory mediators; green, antiinflammatory mediators; gray, data derived from literature (not presented in the manuscript). 


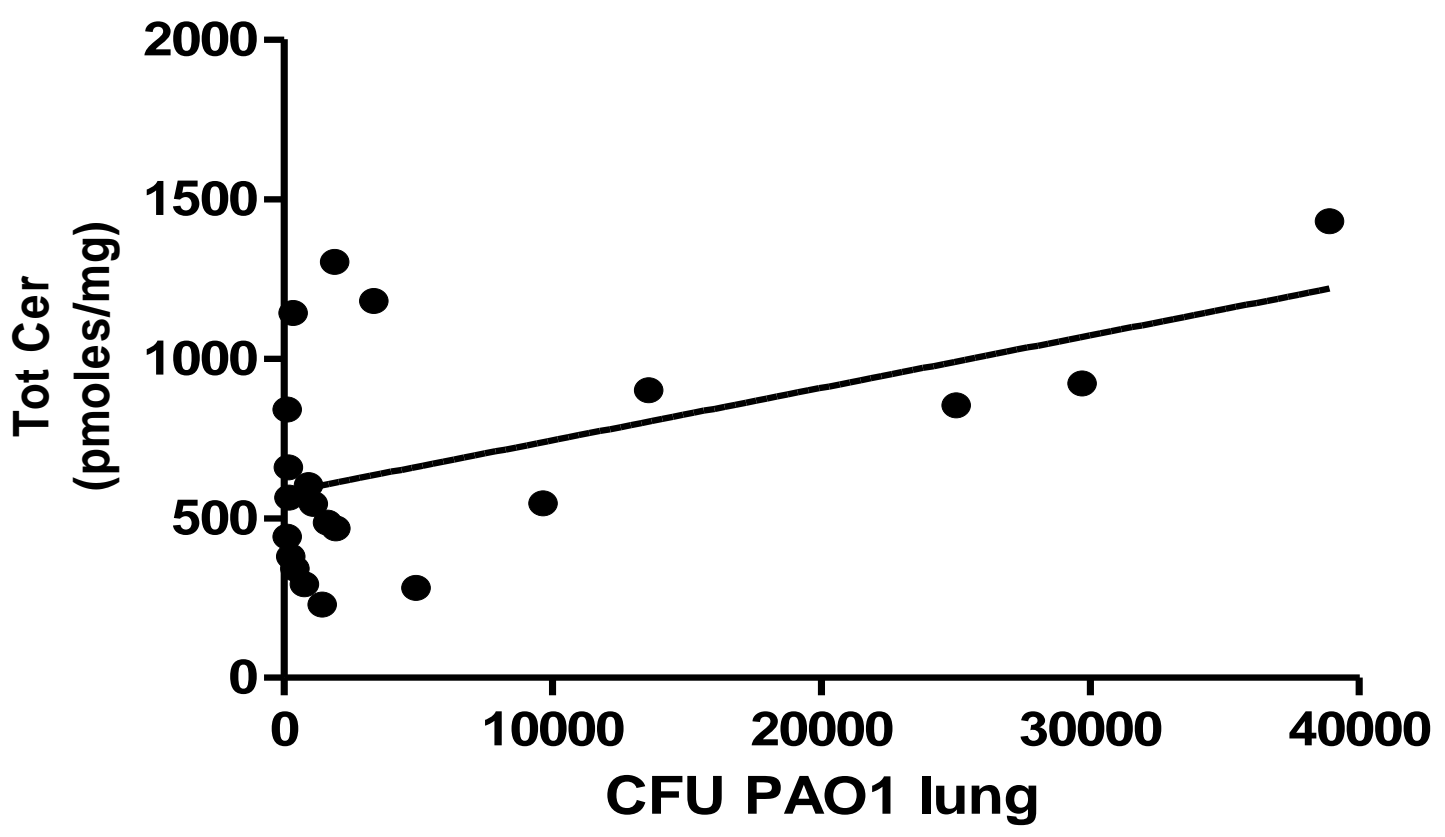


KO

WT

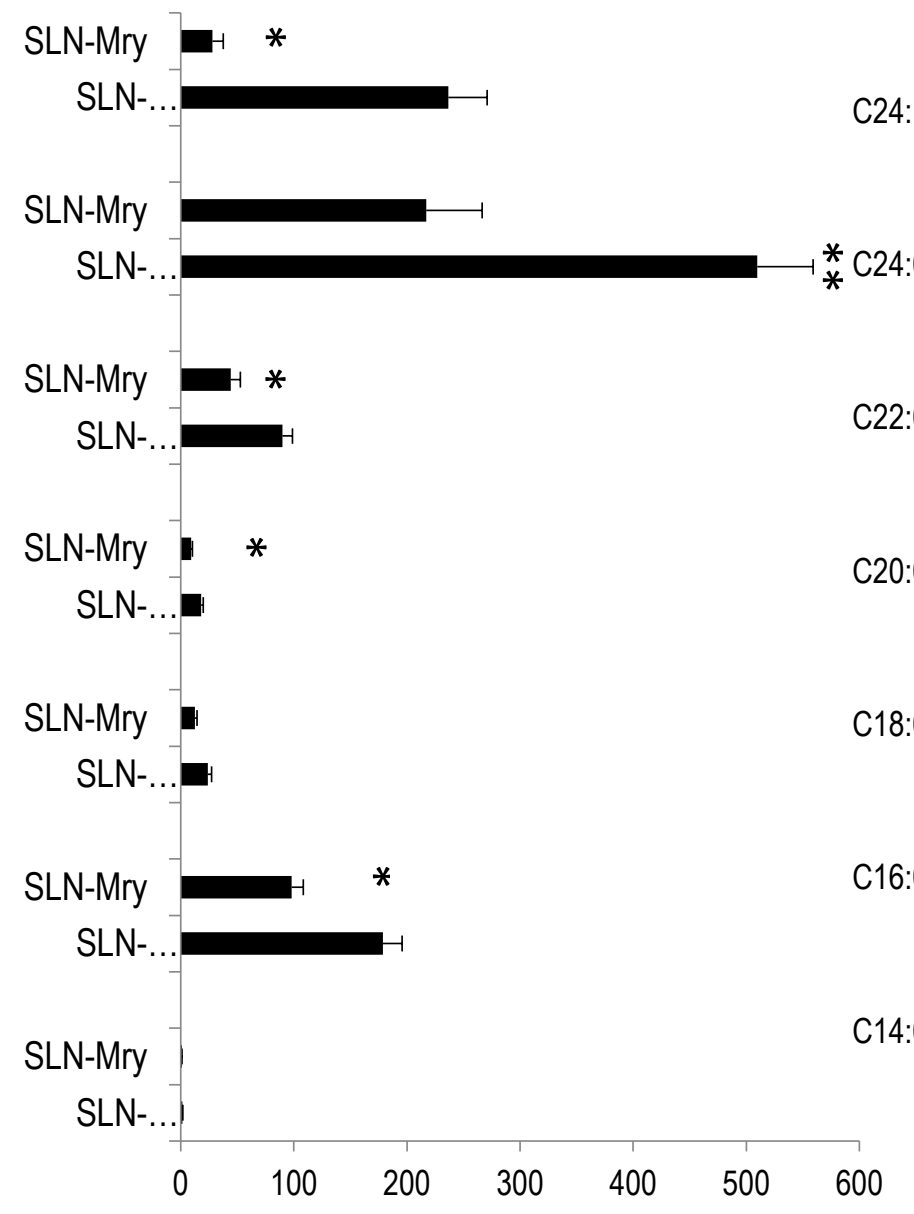

ceramides nanomoles/ proteins micrograms

SLN-Mry

SLN-empty

$\begin{array}{lllllll}0 & 100 & 200 & 300 & 400 & 500 & 600\end{array}$

ceramides nanomoles/ proteins micrograms 


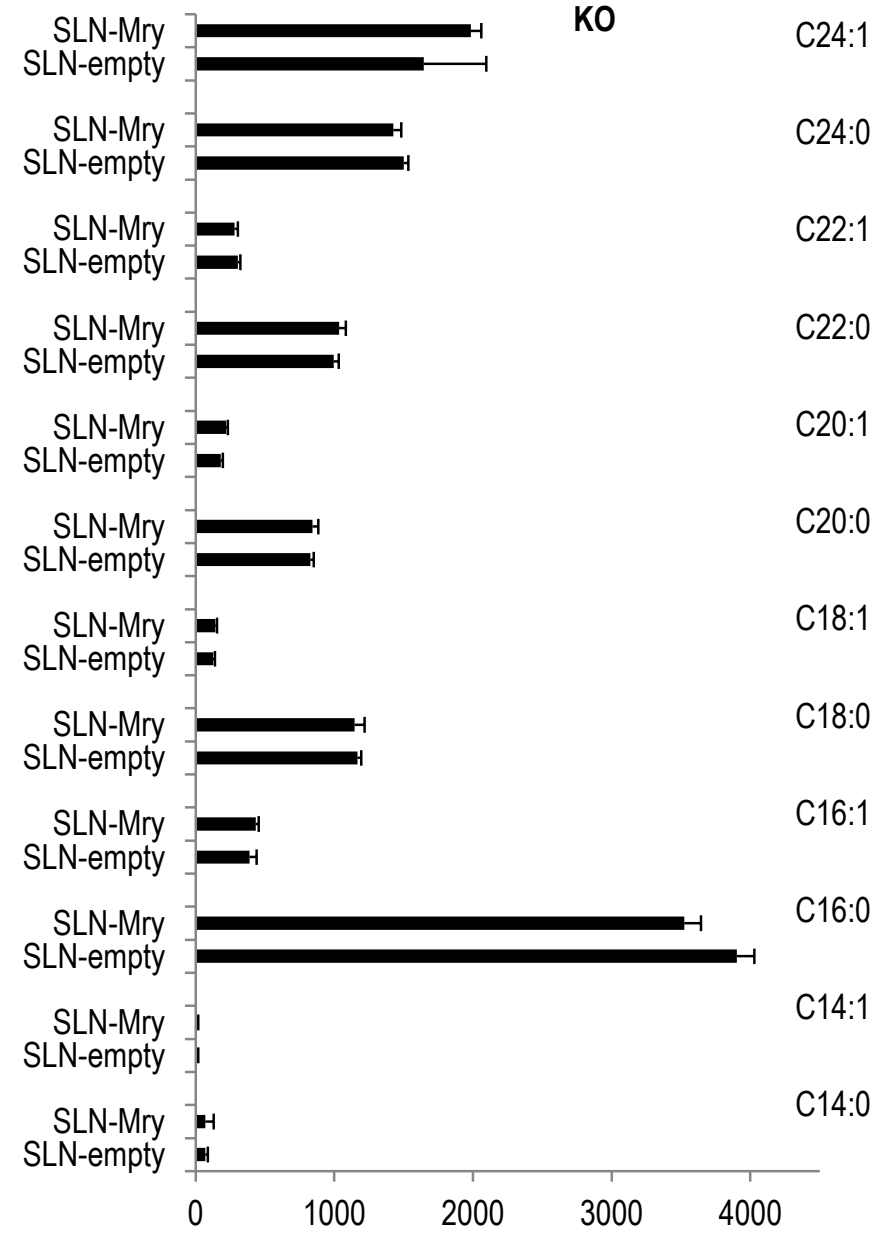

Sphingomyelins nanomoles/proteins micrograms

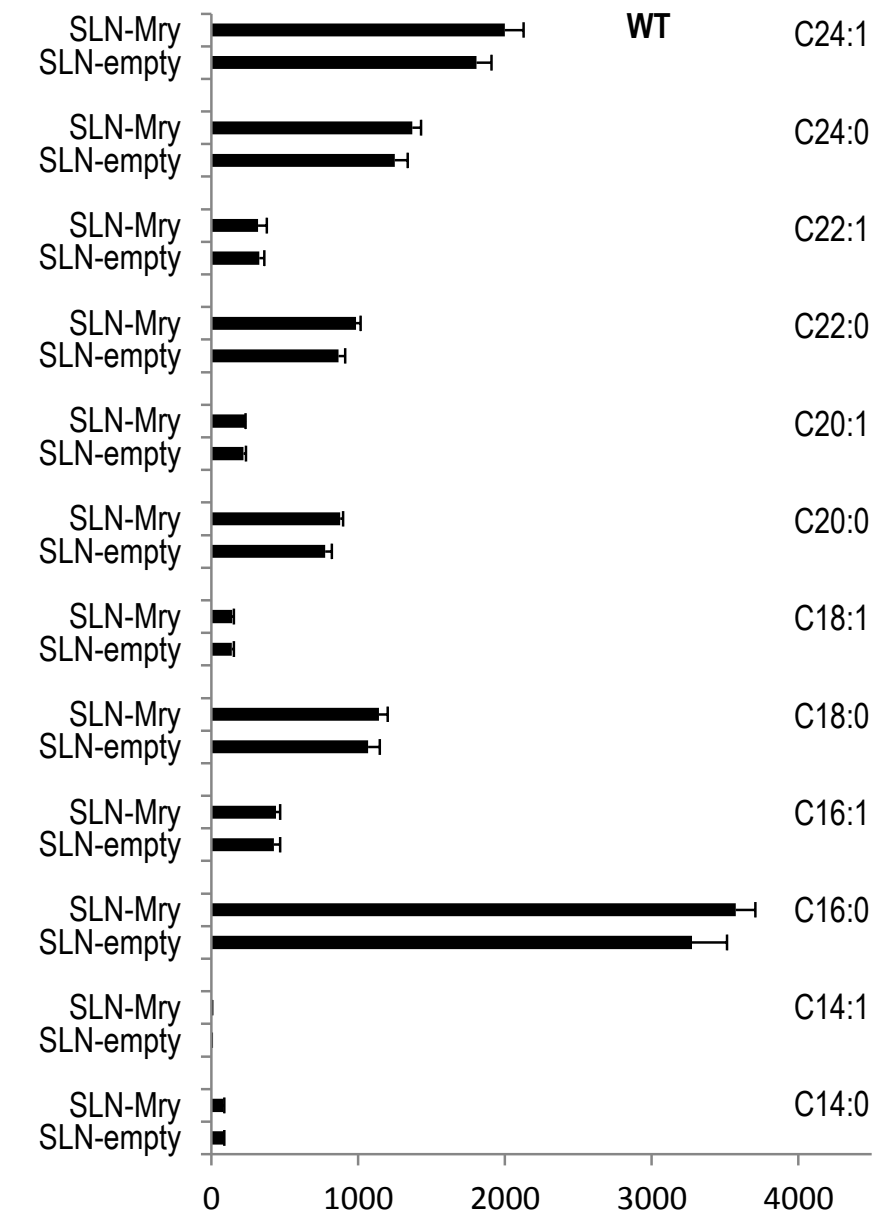

Sphingomyelins nanomoles/proteins micrograms 

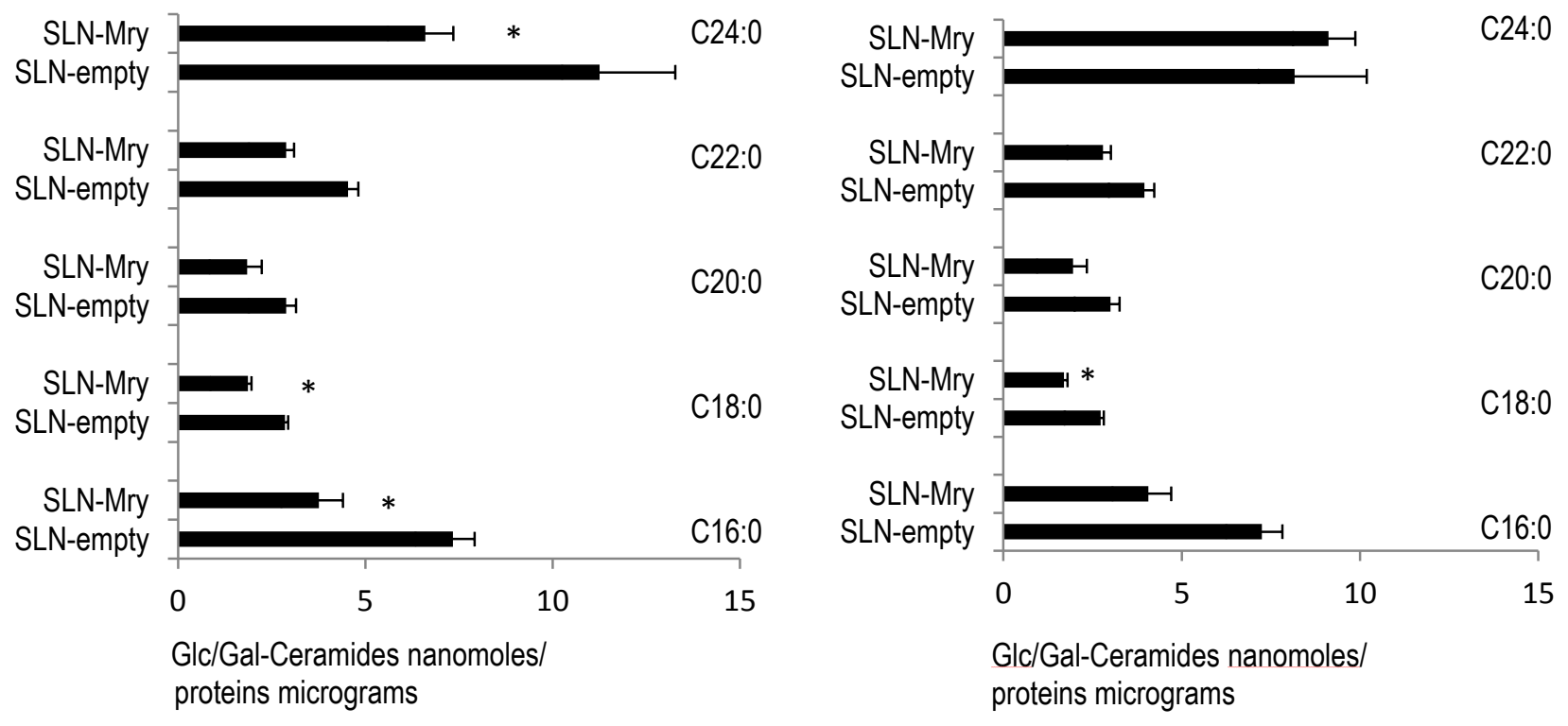


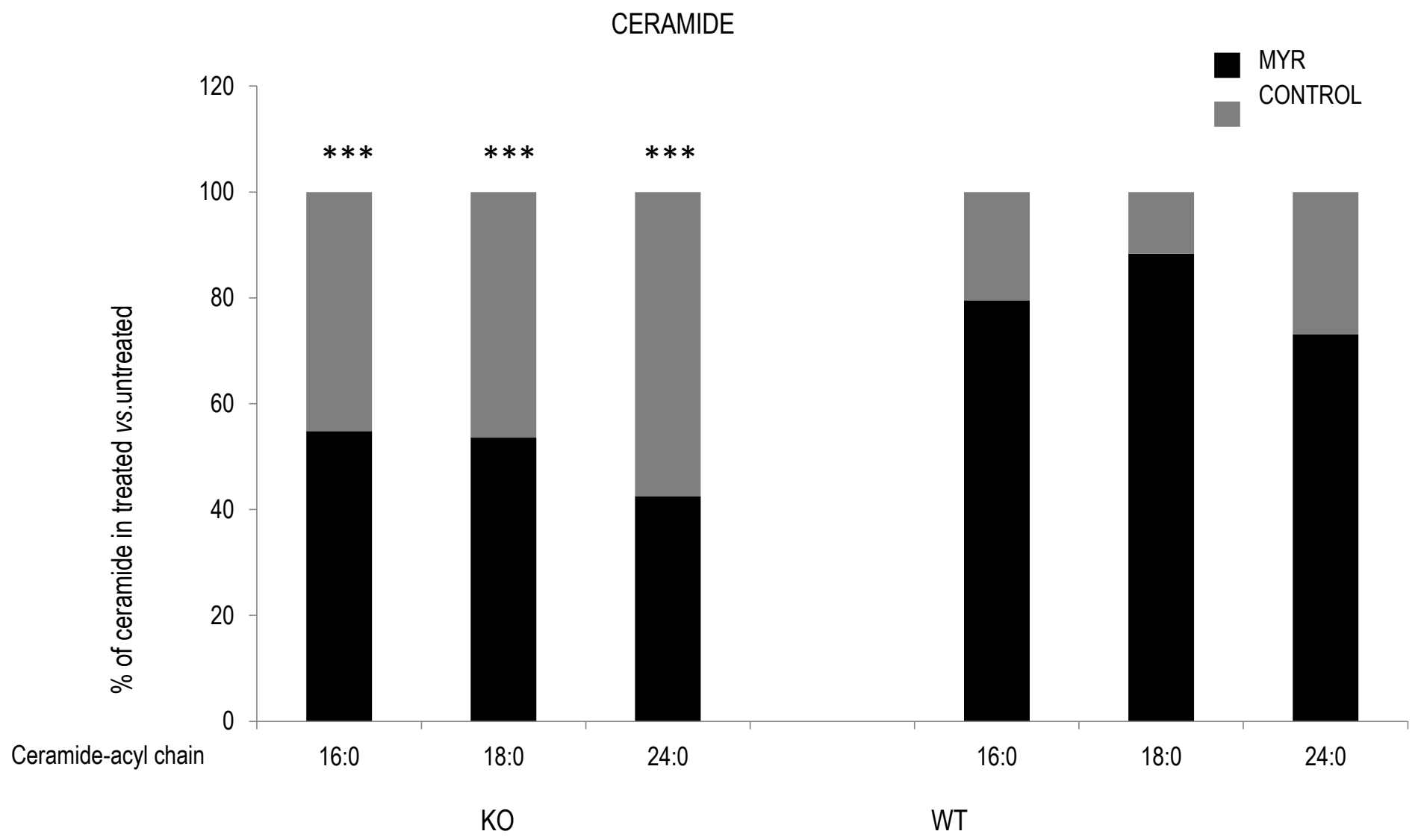


Figure $3 \mathrm{~A}$

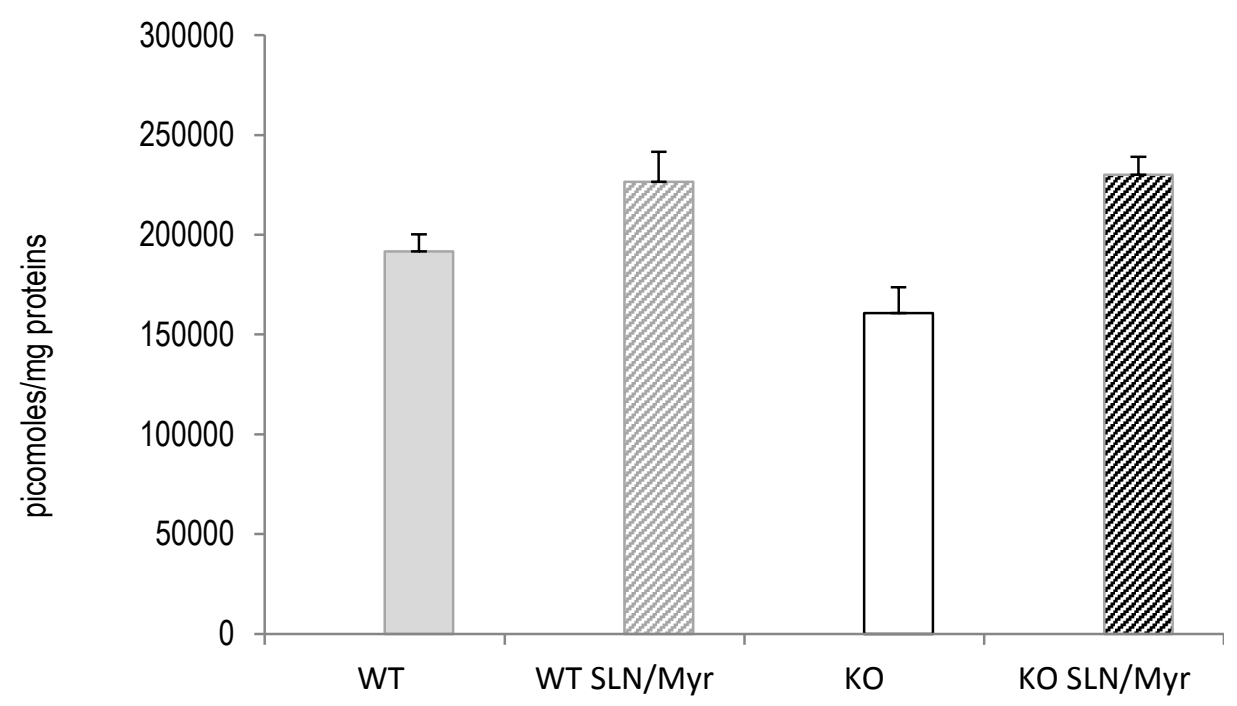




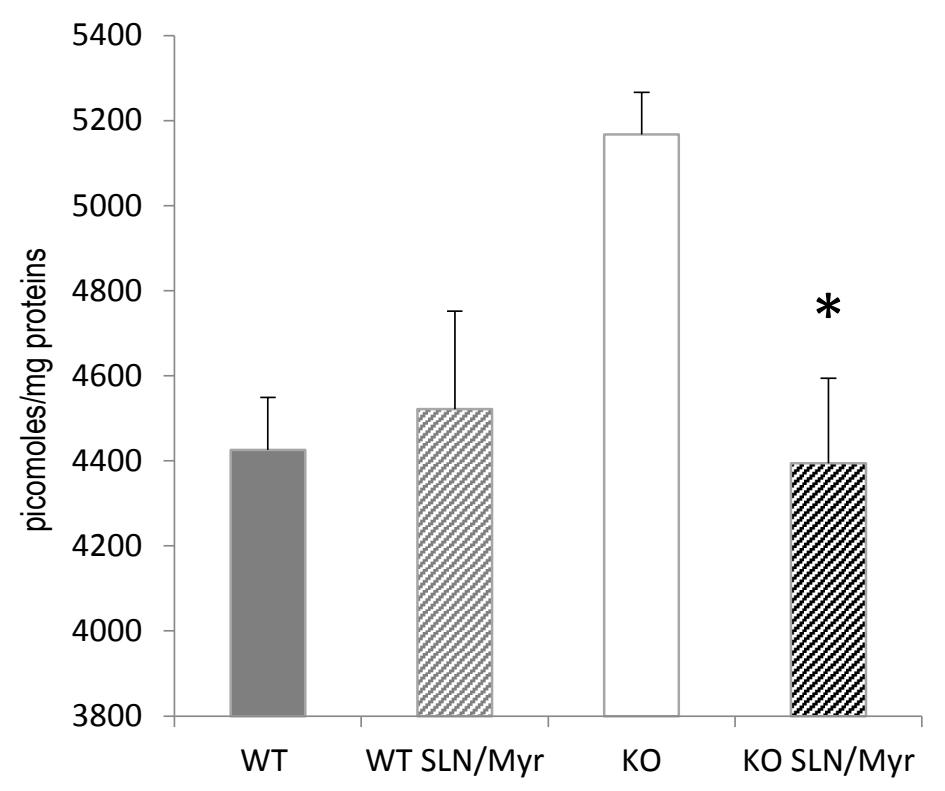


ALVEOLUS
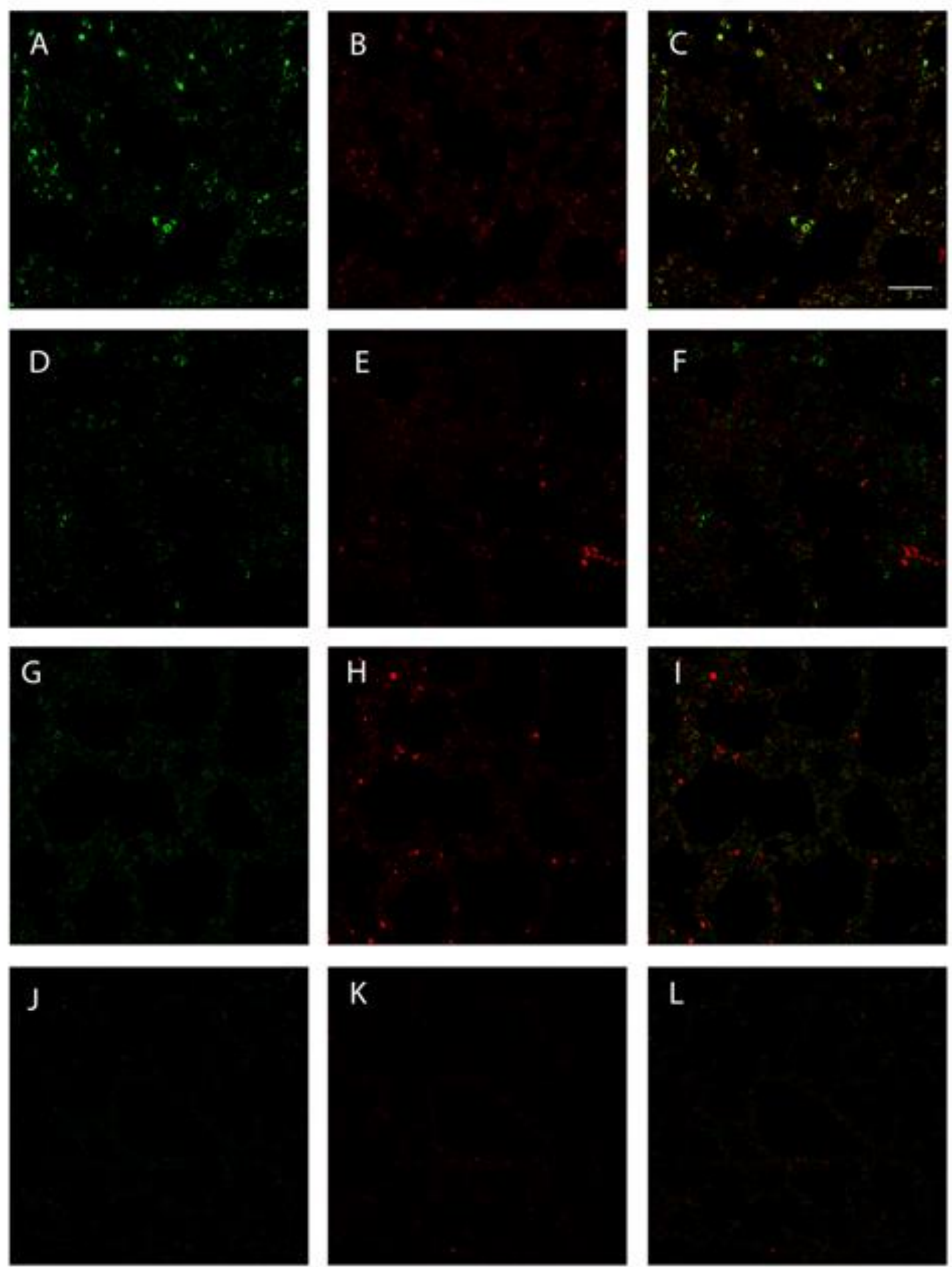
BRONCUS
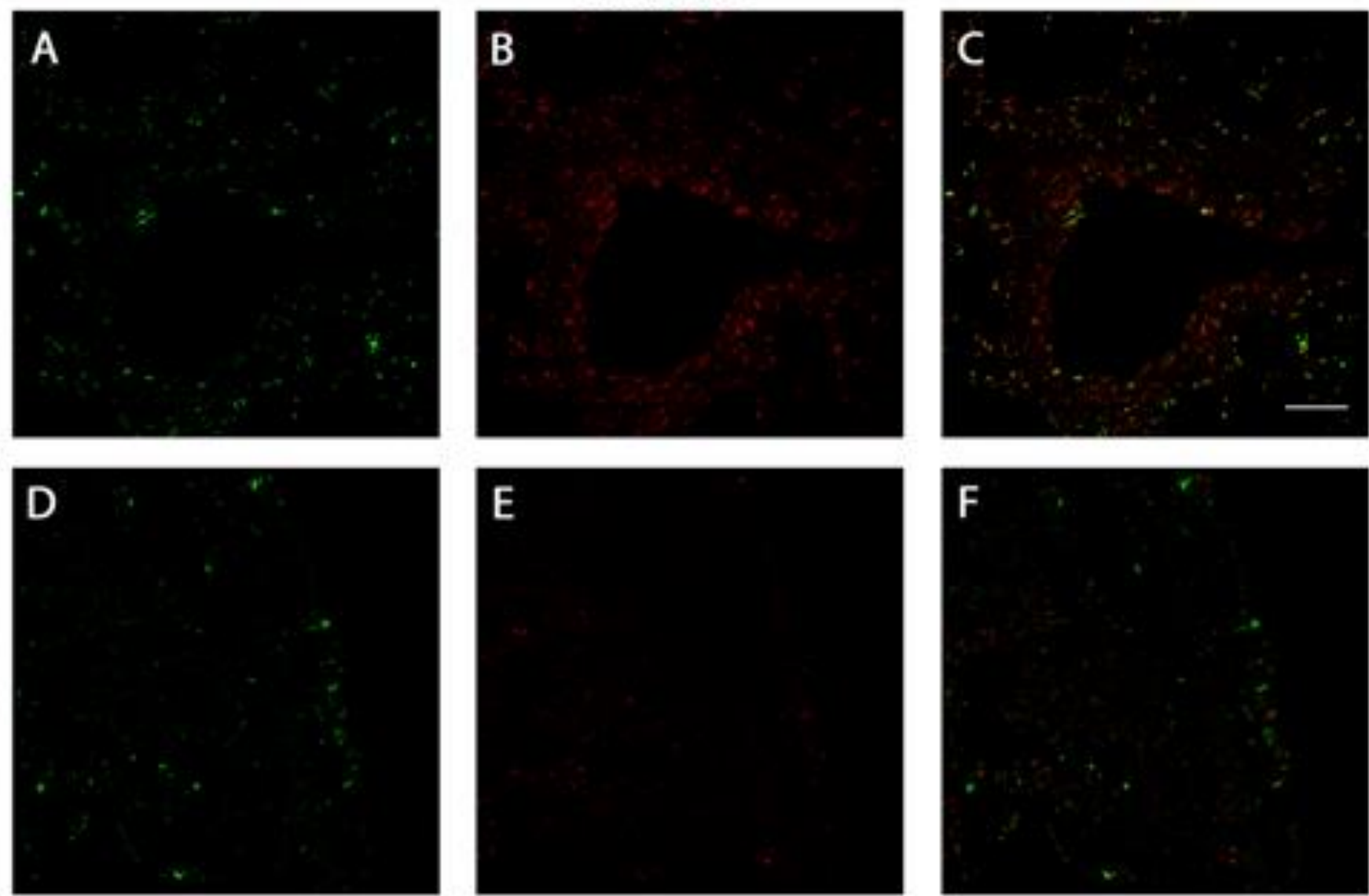

E

F

G

H

I

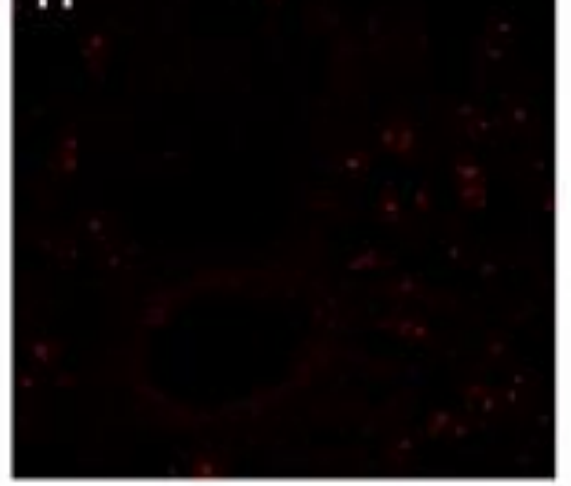

K

L

J

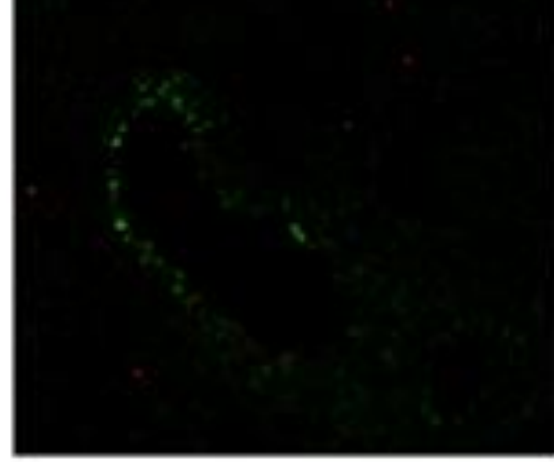




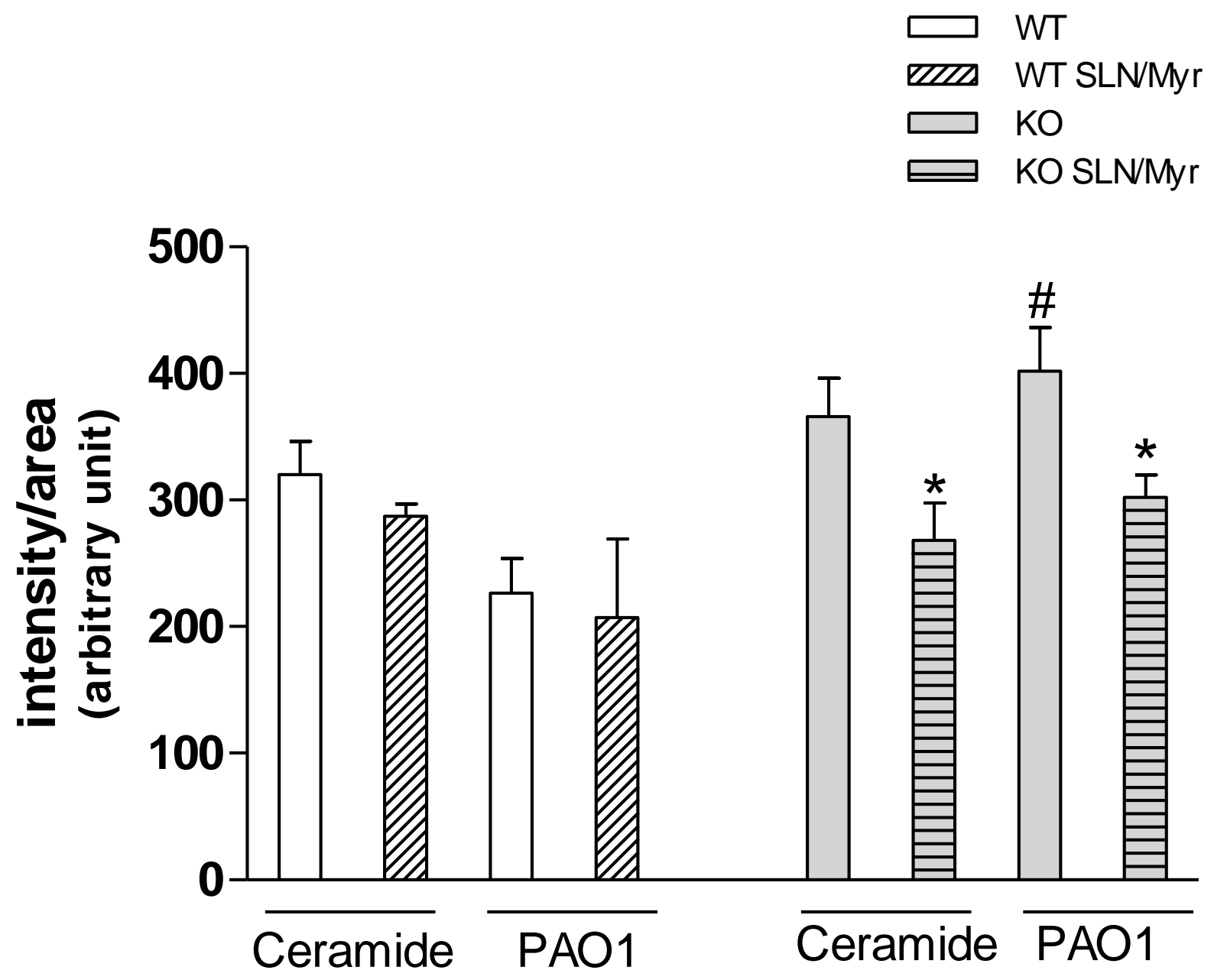



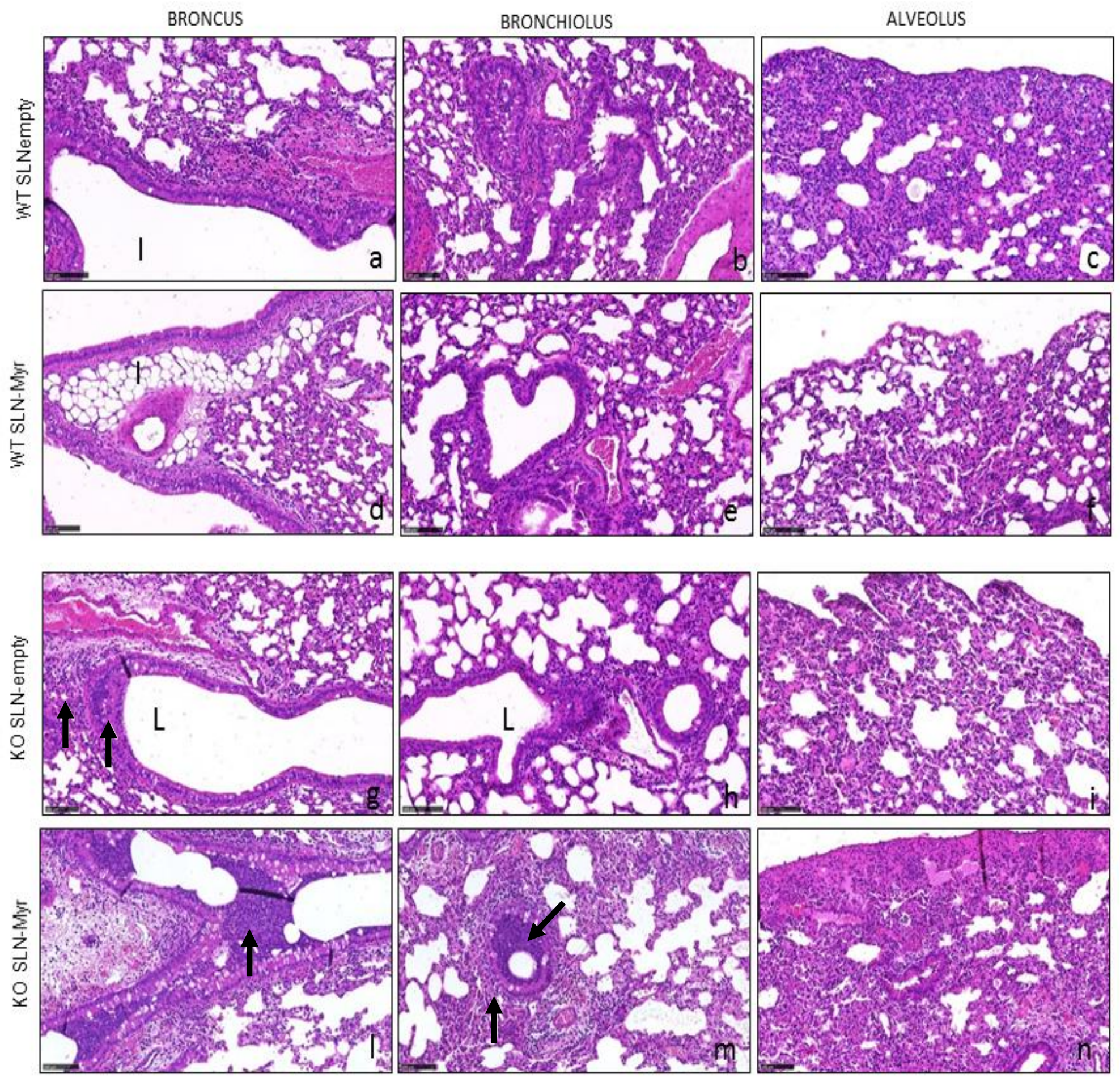


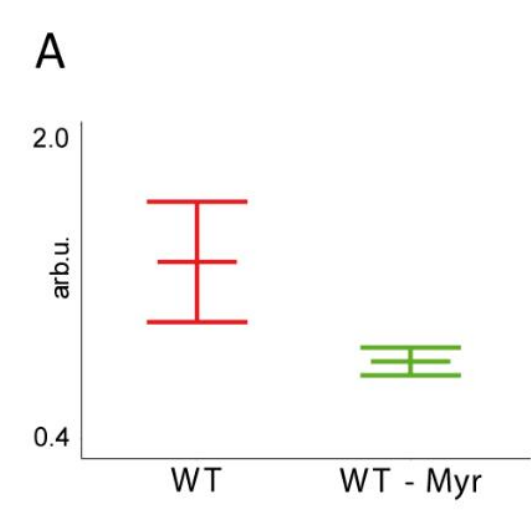

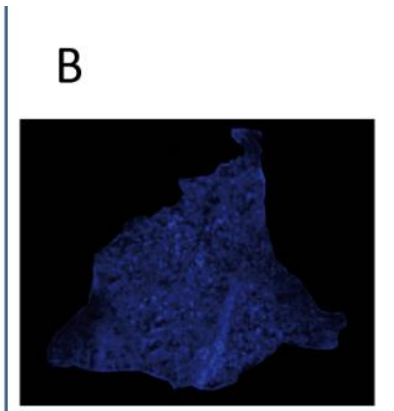

WT

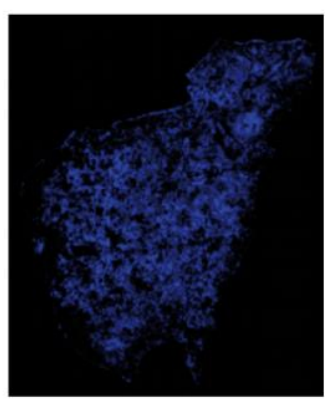

$\mathrm{KO}$

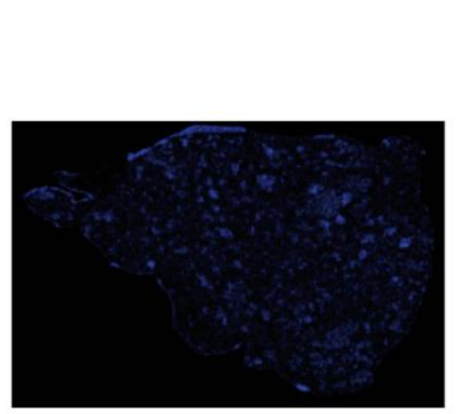

C

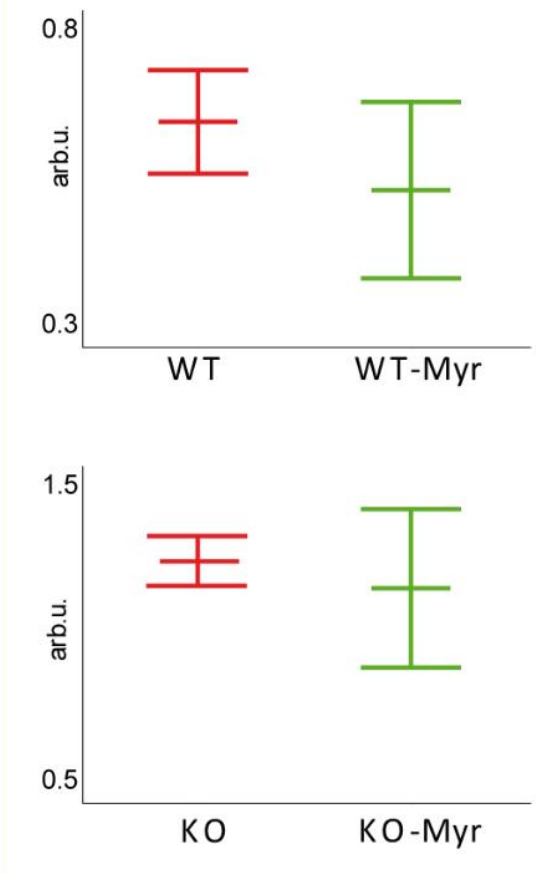

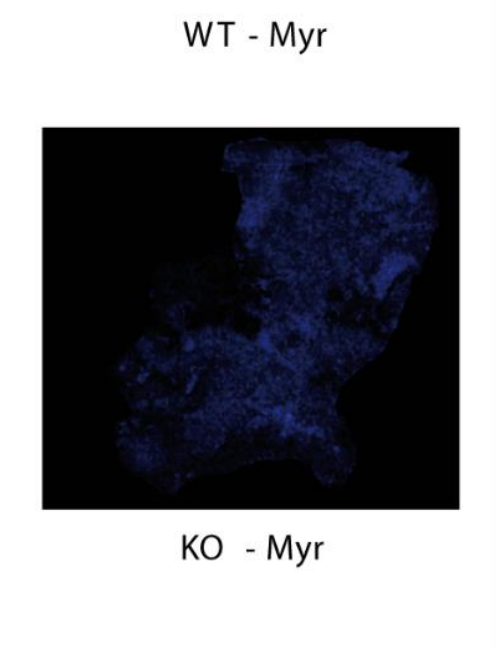

WT - Myr

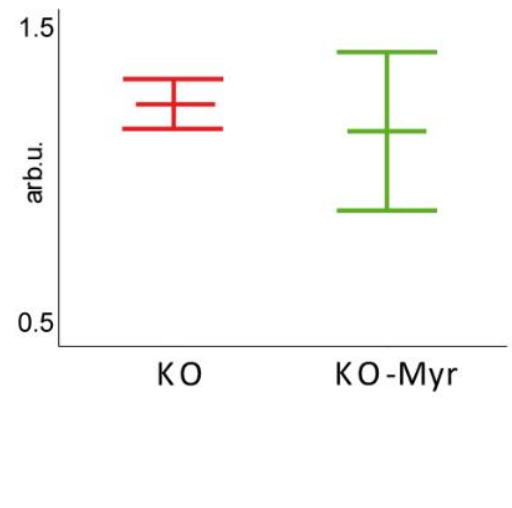

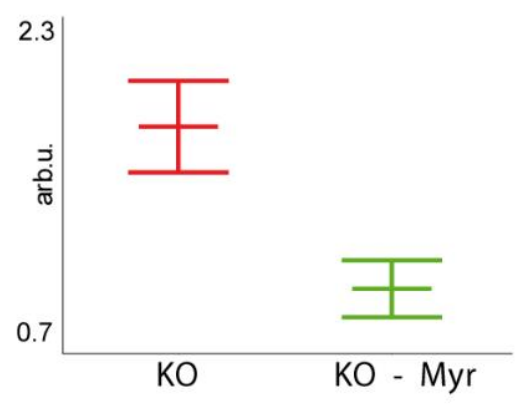




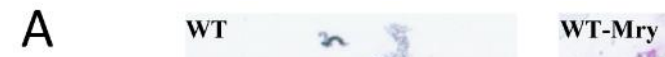

H\&E stain
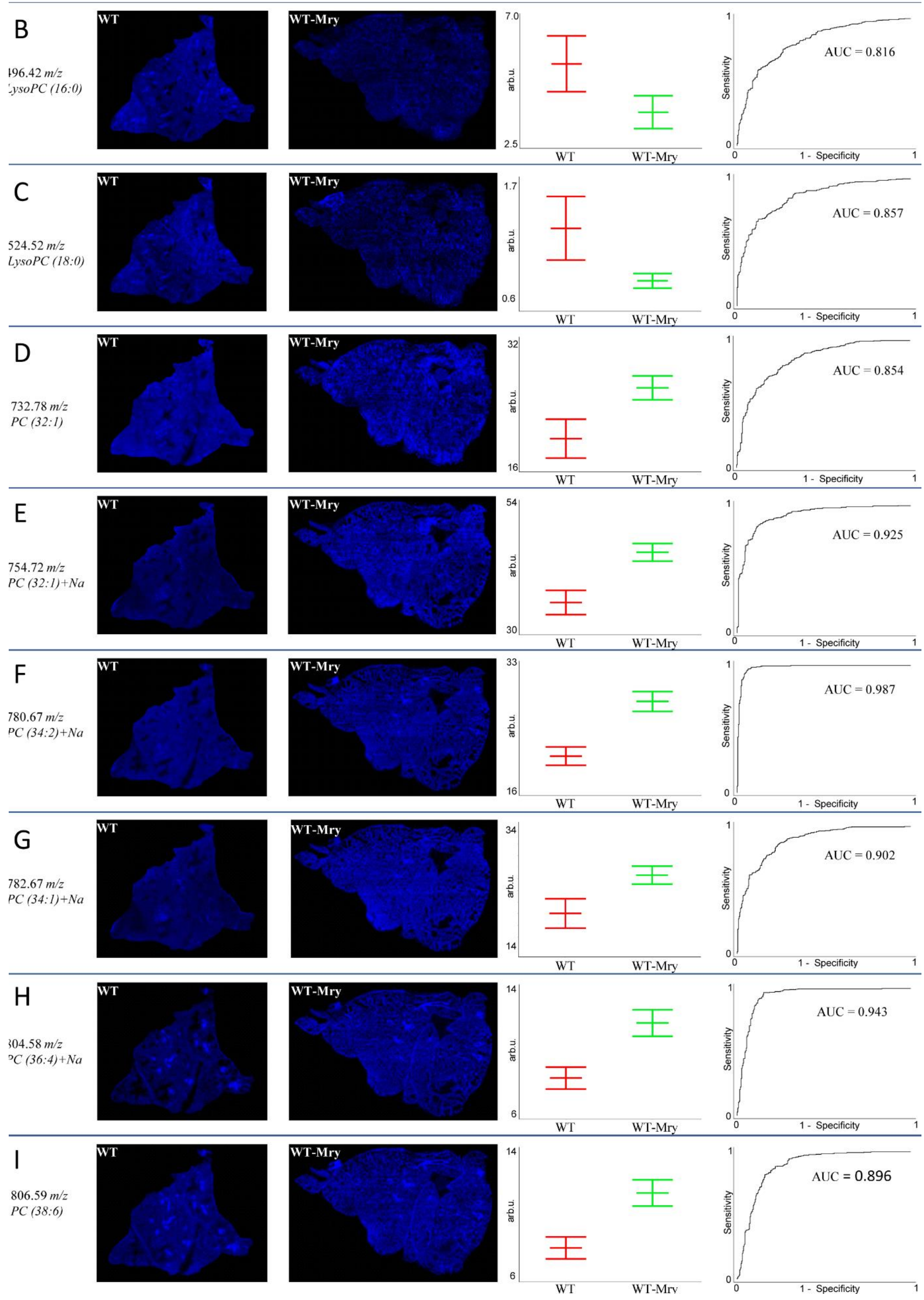

A
Ko
KO-Mry

H\&E stain

$\lim _{2}$
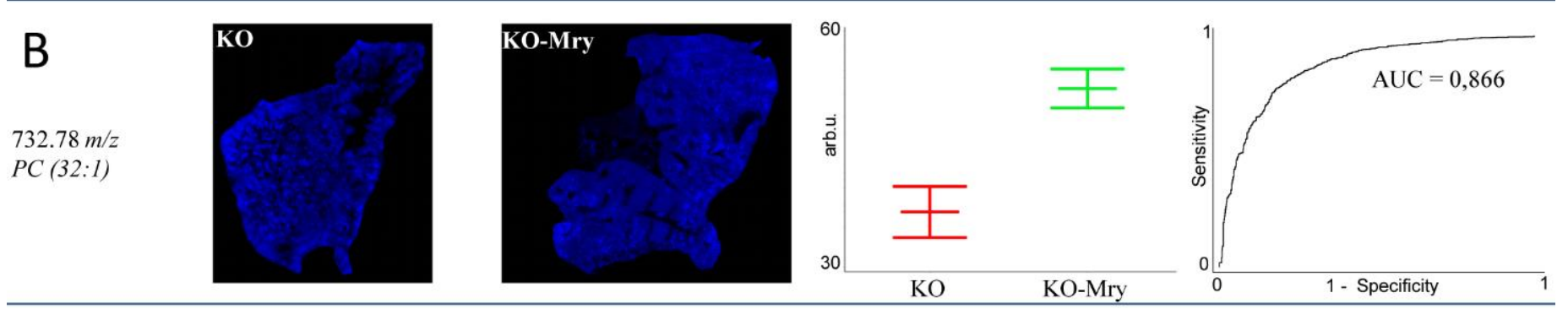

\section{C}

$754.72 \mathrm{~m} / \mathrm{z}$

$\mathrm{PC}(32: 1)+\mathrm{Na}$
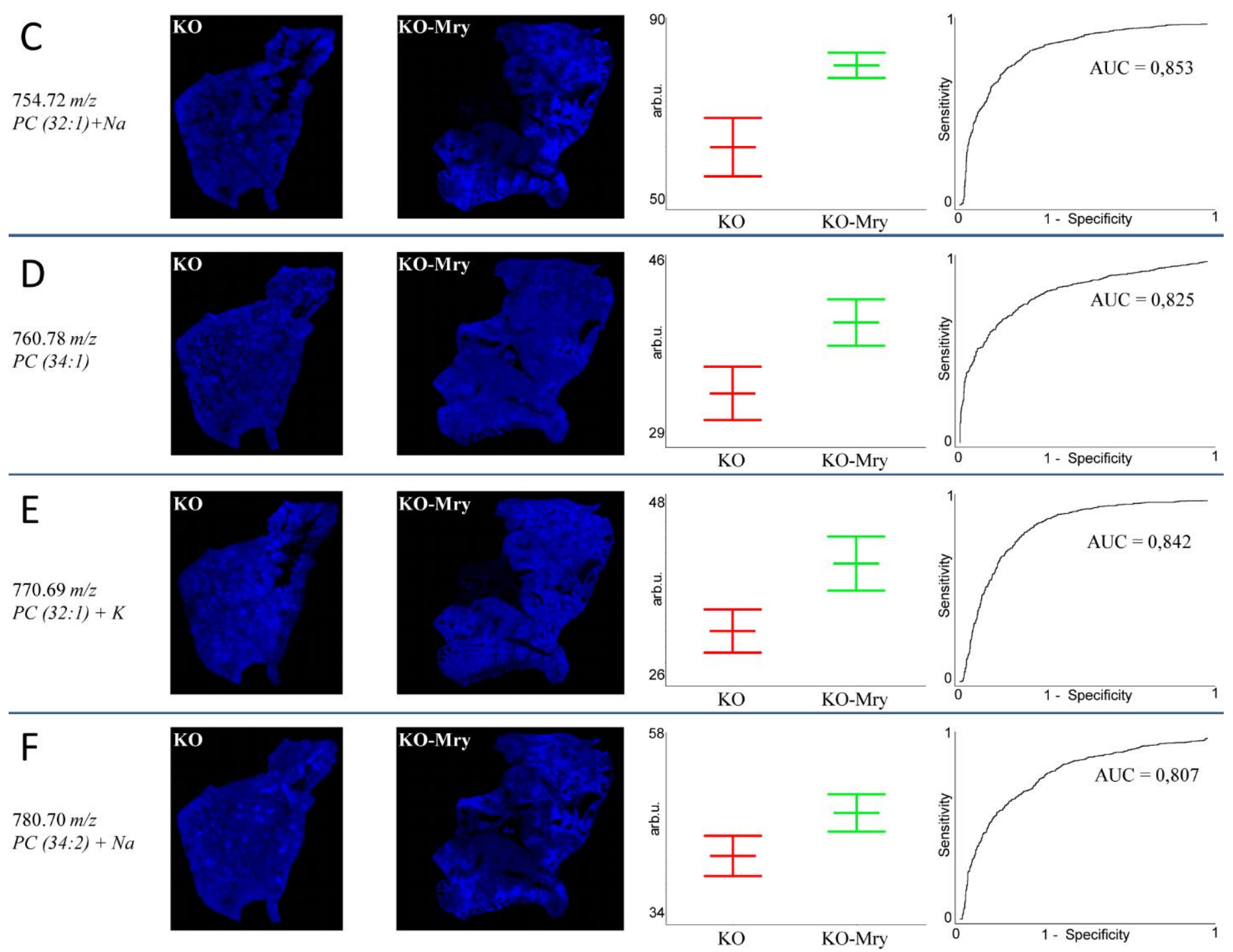
Fatty Acids

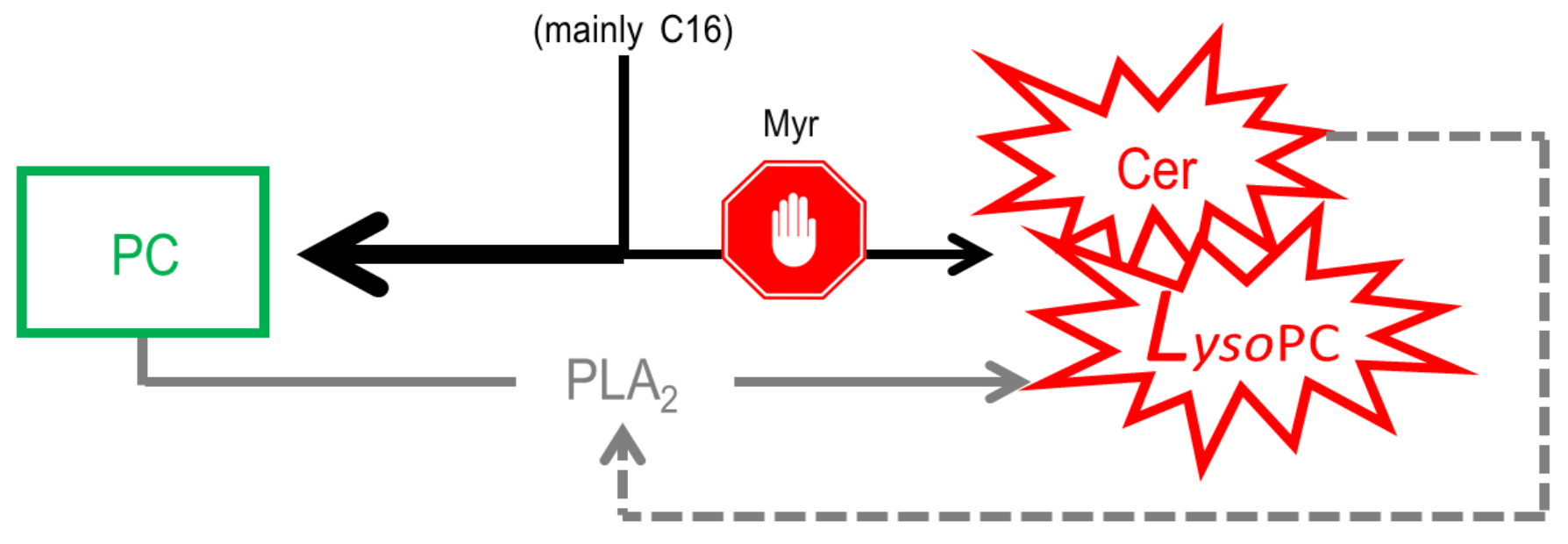

Figure9 


\begin{tabular}{|c|c|c|c|c|c|c|}
\hline Infiltrate evaluation & \multicolumn{2}{|c|}{ Bronchus } & \multicolumn{2}{|c|}{ Brochiolus } & \multicolumn{2}{|c|}{ Alveolus } \\
\hline & $\mathbb{I P}$ & EL & IP & EL & IP & EL \\
\hline WT SLN-empty & - & - & $++D$ & $++D$ & $+D$ & $+D$ \\
\hline WT SLN-Myr & - & - & $+P$ & - & - & - \\
\hline KO SLN-empty & $+D$ & $+D$ & $+++D$ & $+++D$ & $++D$ & $++D$ \\
\hline KO SLN-Myr & $+P$ & - & $++P$ & $+P$ & $+P$ & $P$ \\
\hline
\end{tabular}




\begin{tabular}{|c|c|c|c|c|c|c|c|}
\hline Peak Mass & $\begin{array}{l}\text { Wilcoxon } \\
\text { p-value }\end{array}$ & $\begin{array}{l}\text { WT average } \\
\text { area value }\end{array}$ & $\begin{array}{c}\text { CV(\%) } \\
\text { WT }\end{array}$ & $\begin{array}{l}\text { WT+Myr average } \\
\text { area value }\end{array}$ & $\begin{array}{c}\text { CV(\%) } \\
\text { WT + Myr }\end{array}$ & $\begin{array}{l}\text { ROC } \\
\text { value }\end{array}$ & Assignment \\
\hline 754.72 & 0.00569 & 6.95 & 6.28 & 8.76 & 3.59 & 0.925 & $\mathrm{PC}(32: 1)+\mathrm{Na}$ \\
\hline 772.66 & 0.00569 & 5.89 & 8.82 & 4.39 & 5.59 & 0.940 & $P C(32: 0)+K$ \\
\hline 780.67 & 0.00569 & 4.11 & 5.70 & 5.51 & 4.41 & 0.986 & $\mathrm{PC}(34: 2)+\mathrm{Na}$ \\
\hline 732.78 & 0.00569 & 3.96 & 12.58 & 5.26 & 5.68 & 0.854 & PC (32:1) \\
\hline 782.68 & 0.00569 & 6.29 & 4.65 & 7.45 & 6.73 & 0.902 & $\mathrm{PC}(34: 1)+\mathrm{Na}$ \\
\hline 741.75 & 0.00569 & 2.48 & 8.67 & 1.37 & 13.18 & 0.985 & \\
\hline 675.26 & 0.00569 & 2.29 & 16.85 & 1.37 & 12.76 & 0.914 & \\
\hline 744.71 & 0.00569 & 2.76 & 6.04 & 1.89 & 7.56 & 0.925 & \\
\hline 742.76 & 0.00569 & 2.82 & 8.00 & 2.02 & 10.80 & 0.910 & \\
\hline 828.49 & 0.00569 & 1.63 & 9.20 & 2.37 & 6.67 & 0.953 & \\
\hline 804.58 & 0.00569 & 1.67 & 8.30 & 2.36 & 6.99 & 0.943 & $\mathrm{PC}(36: 4)+\mathrm{Na}$ \\
\hline 806.59 & 0.00569 & 1.97 & 7.16 & 2.65 & 11.41 & 0.896 & PC (38:6) \\
\hline 691.21 & 0.00569 & 0.86 & 9.65 & 0.43 & 8.51 & 0.993 & \\
\hline 752.70 & 0.00569 & 0.62 & 8.36 & 0.99 & 6.54 & 0.963 & \\
\hline 851.47 & 0.00569 & 1.14 & 17.55 & 0.79 & 11.72 & 0.838 & \\
\hline 824.52 & 0.00569 & 1.11 & 8.18 & 0.77 & 8.90 & 0.941 & \\
\hline 534.48 & 0.00569 & 0.61 & 11.74 & 0.28 & 14.61 & 0.984 & \\
\hline 826.52 & 0.00569 & 0.86 & 7.88 & 0.60 & 8.54 & 0.944 & \\
\hline 562.57 & 0.00569 & 0.54 & 13.12 & 0.30 & 13.23 & 0.932 & \\
\hline 802.58 & 0.00569 & 0.81 & 7.89 & 1.04 & 4.16 & 0.895 & \\
\hline 822.51 & 0.00569 & 0.78 & 6.26 & 0.61 & 7.87 & 0.879 & \\
\hline 856.40 & 0.00569 & 0.56 & 6.91 & 0.71 & 9.47 & 0.843 & \\
\hline 635.45 & 0.00569 & 0.23 & 19.80 & 0.11 & 12.98 & 0.969 & \\
\hline 538.17 & 0.00569 & 0.26 & 19.45 & 0.16 & 8.83 & 0.818 & Cer C16 \\
\hline 700.76 & 0.00569 & 0.34 & 4.85 & 0.41 & 7.98 & 0.885 & \\
\hline 666.78 & 0.00569 & 0.3 & 6.76 & 0.24 & 6.83 & 0.835 & \\
\hline 524.52 & 0.0187 & 0.72 & 19.01 & 0.45 & 13.43 & 0.857 & LysoPC (18:0) \\
\hline 496.42 & 0.0292 & 1.05 & 17.98 & 0.72 & 14.89 & 0.816 & LysoPC (16:0) \\
\hline 848.41 & 0.0292 & 0.52 & 7.78 & 0.43 & 9.95 & 0.803 & \\
\hline 672.72 & 0.0466 & 0.41 & 11.00 & 0.53 & 16.54 & 0.808 & \\
\hline
\end{tabular}




\begin{tabular}{cccccccc}
\hline Peak Mass & $\begin{array}{c}\text { Wilcoxon } \\
\text { p-value }\end{array}$ & $\begin{array}{c}\text { KO average } \\
\text { area value }\end{array}$ & $\begin{array}{c}\mathbf{C V}(\%) \\
\text { KO }\end{array}$ & $\begin{array}{c}\text { KO }+ \text { Myr } \\
\text { average area } \\
\text { value }\end{array}$ & $\begin{array}{c}\text { CV(\%) } \\
\text { KO + Myr }\end{array}$ & $\begin{array}{c}\text { ROC } \\
\text { value }\end{array}$ & Assignment \\
\hline 754.75 & 0.00222 & 8.43 & 10.38 & 10.88 & 3.52 & 0.853 & PC $(32: 1)+\mathrm{Na}$ \\
732.82 & 0.00222 & 5.01 & 8.76 & 7.12 & 4.70 & 0.866 & $\mathrm{PC}(32: 1)$ \\
567.27 & 0.00222 & 2.06 & 20.53 & 0.72 & 17.41 & 0.995 & \\
770.69 & 0.00222 & 4.33 & 8.59 & 5.50 & 8.49 & 0.842 & $\mathrm{PC}(32: 1)+\mathrm{K}$ \\
760.78 & 0.00222 & 4.50 & 7.91 & 5.44 & 5.70 & 0.825 & $\mathrm{PC}(34: 1)$ \\
583.27 & 0.00222 & 1.03 & 16.52 & 0.47 & 14.20 & 0.976 & \\
588.28 & 0.00222 & 0.32 & 17.25 & 0.14 & 12.35 & 0.979 & \\
572.28 & 0.00222 & 0.31 & 16.47 & 0.17 & 8.84 & 0.961 & \\
538.20 & 0.00222 & 0.24 & 13.43 & 0.13 & 15.90 & 0.908 & $\mathrm{Cer} \mathrm{C} 16$ \\
577.82 & 0.00222 & 0.16 & 7.94 & 0.26 & 10.38 & 0.919 & \\
627.89 & 0.00222 & 0.16 & 12.37 & 0.25 & 11.47 & 0.896 & \\
511.17 & 0.00222 & 0.18 & 15.03 & 0.11 & 16.95 & 0.881 & \\
780.70 & 0.0114 & 5.63 & 0.36 & 6.41 & 0.34 & 0.807 & $\mathrm{PC}(34: 2)+\mathrm{Na}$ \\
650.79 & 0.0114 & 0.29 & 19.73 & 0.19 & 15.14 & 0.808 & \\
\hline
\end{tabular}

